\title{
Bôcher Contractions of Conformally Superintegrable Laplace Equations ${ }^{\star}$
}

\author{
Ernest G. KALNINS ${ }^{\dagger}$, Willard MILLER Jr. ${ }^{\ddagger}$ and Eyal SUBAG ${ }^{\S}$ \\ $\dagger$ Department of Mathematics, University of Waikato, Hamilton, New Zealand \\ E-mail: math0236@math.waikato.ac.nz \\ URL: http://www.math.waikato.ac.nz \\ $\ddagger$ School of Mathematics, University of Minnesota, Minneapolis, Minnesota, 55455, USA \\ E-mail: miller@ima.umn.edu \\ URL: http://www.ima.umn.edu/ miller/ \\ $\S$ Department of Mathematics, Pennsylvania State University, State College, \\ Pennsylvania, 16802 USA \\ E-mail:eus25@psu.edu
}

Received January 24, 2016, in final form April 11, 2016; Published online April 19, 2016

http://dx.doi.org/10.3842/SIGMA.2016.038

\begin{abstract}
The explicit solvability of quantum superintegrable systems is due to symmetry, but the symmetry is often "hidden". The symmetry generators of 2nd order superintegrable systems in 2 dimensions close under commutation to define quadratic algebras, a generalization of Lie algebras. Distinct systems on constant curvature spaces are related by geometric limits, induced by generalized Inönü-Wigner Lie algebra contractions of the symmetry algebras of the underlying spaces. These have physical/geometric implications, such as the Askey scheme for hypergeometric orthogonal polynomials. However, the limits have no satisfactory Lie algebra contraction interpretations for underlying spaces with 1- or 0-dimensional Lie algebras. We show that these systems can be best understood by transforming them to Laplace conformally superintegrable systems, with flat space conformal symmetry group $\mathrm{SO}(4, \mathbb{C})$, and using ideas introduced in the 1894 thesis of Bôcher to study separable solutions of the wave equation in terms of roots of quadratic forms. We show that Bôcher's prescription for coalescing roots of these forms induces contractions of the conformal algebra $\mathfrak{s o}(4, \mathbb{C})$ to itself and yields a mechanism for classifying all Helmholtz superintegrable systems and their limits. In the paper [Acta Polytechnica, to appear, arXiv:1510.09067], we announced our main findings. This paper provides the proofs and more details.
\end{abstract}

Key words: conformal superintegrability; contractions; Laplace equations

2010 Mathematics Subject Classification: 81R05; 81R12; 33C45

\section{Introduction}

A quantum (or Helmholtz) superintegrable system is an integrable Hamiltonian system on an $n$-dimensional Riemannian/pseudo-Riemannian manifold with potential: $H=\Delta_{n}+V$ that admits $2 n-1$ algebraically independent partial differential operators $L_{j}$ commuting with $H$, the maximum possible. $\left[H, L_{j}\right]=0, j=1,2, \ldots, 2 n-1$. Superintegrability captures the properties of quantum Hamiltonian systems that allow the Schrödinger eigenvalue problem (or Helmholtz equation) $H \Psi=E \Psi$ to be solved exactly, analytically and algebraically [6, 7, 32, 36, 37]. A system is of order $K$ if the maximum order of the symmetry operators, other than $H$, is $K$.

\footnotetext{
${ }^{\star}$ This paper is a contribution to the Special Issue on Orthogonal Polynomials, Special Functions and Applications. The full collection is available at http://www.emis.de/journals/SIGMA/OPSFA2015.html
} 
For $n=2, K=1,2$ all systems are known, e.g., [5, 12, 13, 14, 15, 16, 17]. For $K=1$ the symmetry algebras are just Lie algebras.

We review quickly the facts for free 2 nd order superintegrable systems (i.e., no potential, $K=2$ ) in the case $n=2,2 n-1=3$. The complex spaces with Laplace-Beltrami operators admitting at least three 2nd order symmetries were classified by Koenigs [29]. They are:

- the two constant curvature spaces (flat space and the complex sphere), six linearly independent 2nd order symmetries and three 1st order symmetries,

- the four Darboux spaces (one of which, D4, contains a parameter), four 2nd order symmetries and one 1st order symmetry, see Section 1.1 and [19],

- 6 families of 4-parameter Koenigs spaces. No 1st order symmetries, see Section 1.1.

For 2nd order systems with non-constant potential, $K=2$, the generating symmetry operators of each system close under commutation to determine a quadratic algebra, and the irreducible representations of this algebra determine the eigenvalues of $H$ and their multiplicities. Here we consider only the nondegenerate superintegrable systems. Those with 4-parameter potentials (including the additive constant) (the maximum possible):

$$
V(\mathbf{x})=a_{1} V_{(1)}(\mathbf{x})+a_{2} V_{(2)}(\mathbf{x})+a_{3} V_{(3)}(\mathbf{x})+a_{4},
$$

where $\left\{V_{(1)}(\mathbf{x}), V_{(2)}(\mathbf{x}), V_{(3)}(\mathbf{x}), 1\right\}$ is a linearly independent set. For these the symmetry algebra generated by $H, L_{1}, L_{2}$ always closes under commutation and gives the following quadratic algebra structure: Define the $3 r d$ order commutator $R$ by $R=\left[L_{1}, L_{2}\right]$. Then

$$
\begin{aligned}
{\left[L_{j}, R\right]=} & A_{1}^{(j)} L_{1}^{2}+A_{2}^{(j)} L_{2}^{2}+A_{3}^{(j)} H^{2}+A_{4}^{(j)}\left\{L_{1}, L_{2}\right\}+A_{5}^{(j)} H L_{1}+A_{6}^{(j)} H L_{2} \\
& +A_{7}^{(j)} L_{1}+A_{8}^{(j)} L_{2}+A_{9}^{(j)} H+A_{10}^{(j)} \\
R^{2}= & b_{1} L_{1}^{3}+b_{2} L_{2}^{3}+b_{3} H^{3}+b_{4}\left\{L_{1}^{2}, L_{2}\right\}+b_{5}\left\{L_{1}, L_{2}^{2}\right\}+b_{6} L_{1} L_{2} L_{1}+b_{7} L_{2} L_{1} L_{2} \\
& +b_{8} H\left\{L_{1}, L_{2}\right\}+b_{9} H L_{1}^{2}+b_{10} H L_{2}^{2}+b_{11} H^{2} L_{1}+b_{12} H^{2} L_{2}+b_{13} L_{1}^{2}+b_{14} L_{2}^{2} \\
& +b_{15}\left\{L_{1}, L_{2}\right\}+b_{16} H L_{1}+b_{17} H L_{2}+b_{18} H^{2}+b_{19} L_{1}+b_{20} L_{2}+b_{21} H+b_{22},
\end{aligned}
$$

where $\left\{L_{1}, L_{2}\right\}=L_{1} L_{2}+L_{2} L_{1}$ and the $A_{i}^{(j)}, b_{k}$ are constants, different for each algebra.

All 2nd order 2D superintegrable systems with potential and their quadratic algebras are known. There are 33 nondegenerate systems, on a variety of manifolds, see Section 1.1 (just manifolds classified by Koenigs), where the numbering for constant curvature systems is taken from [20] (the numbers are not always consecutive because the lists in [20] also include degenerate systems). Under the Stäckel transform (we discuss this in Section 2.1) these systems divide into 8 equivalence classes with representatives on flat space and the 2 -sphere, see [30] and Section 3.16.

\subsection{The Helmholtz nondegenerate superintegrable systems}

Flat space systems: $H \Psi=\left(\partial_{x}^{2}+\partial_{y}^{2}+V\right) \Psi=E \Psi$.

1. $E 1: V=\alpha\left(x^{2}+y^{2}\right)+\frac{\beta}{x^{2}}+\frac{\gamma}{y^{2}}$,

2. $E 2: V=\alpha\left(4 x^{2}+y^{2}\right)+\beta x+\frac{\gamma}{y^{2}}$,

3. $E 3^{\prime}: V=\alpha\left(x^{2}+y^{2}\right)+\beta x+\gamma y$,

4. $E 7: V=\frac{\alpha(x+i y)}{\sqrt{(x+i y)^{2}-b}}+\frac{\beta(x-i y)}{\sqrt{(x+i y)^{2}-b}\left(x+i y+\sqrt{(x+i y)^{2}-b}\right)^{2}}+\gamma\left(x^{2}+y^{2}\right)$,

5. $E 8: V=\frac{\alpha(x-i y)}{(x+i y)^{3}}+\frac{\beta}{(x+i y)^{2}}+\gamma\left(x^{2}+y^{2}\right)$, 
6. $E 9: V=\frac{\alpha}{\sqrt{x+i y}}+\beta y+\frac{\gamma(x+2 i y)}{\sqrt{x+i y}}$,

7. $E 10: V=\alpha(x-i y)+\beta\left(x+i y-\frac{3}{2}(x-i y)^{2}\right)+\gamma\left(x^{2}+y^{2}-\frac{1}{2}(x-i y)^{3}\right)$,

8. $E 11: V=\alpha(x-i y)+\frac{\beta(x-i y)}{\sqrt{x+i y}}+\frac{\gamma}{\sqrt{x+i y}}$,

9. $E 15: V=f(x-i y)$, where $f$ is arbitrary (the exceptional case, characterized by the fact that the symmetry generators are functionally linearly dependent $[12,13,14,15,16,17$, 20]),

10. $E 16: V=\frac{1}{\sqrt{x^{2}+y^{2}}}\left(\alpha+\frac{\beta}{y+\sqrt{x^{2}+y^{2}}}+\frac{\gamma}{y-\sqrt{x^{2}+y^{2}}}\right)$,

11. $E 17: V=\frac{\alpha}{\sqrt{x^{2}+y^{2}}}+\frac{\beta}{(x+i y)^{2}}+\frac{\gamma}{(x+i y) \sqrt{x^{2}+y^{2}}}$,

12. $E 19: \quad V=\frac{\alpha(x+i y)}{\sqrt{(x+i y)^{2}-4}}+\frac{\beta}{\sqrt{(x-i y)(x+i y+2)}}+\frac{\gamma}{\sqrt{(x-i y)(x+i y-2)}}$,

13. $E 20: V=\frac{1}{\sqrt{x^{2}+y^{2}}}\left(\alpha+\beta \sqrt{x+\sqrt{x^{2}+y^{2}}}+\gamma \sqrt{x-\sqrt{x^{2}+y^{2}}}\right)$.

Systems on the complex 2-sphere: $H \Psi=\left(J_{23}^{2}+J_{13}^{2}+J_{12}^{2}+V\right) \Psi=E \Psi$. Here, $J_{k \ell}=$ $s_{k} \partial_{s_{\ell}}-s_{\ell} \partial_{s_{k}}$ and $s_{1}^{2}+s_{2}^{2}+s_{3}^{2}=1$.

1. $S 1: V=\frac{\alpha}{\left(s_{1}+i s_{2}\right)^{2}}+\frac{\beta s_{3}}{\left(s_{1}+i s_{2}\right)^{2}}+\frac{\gamma\left(1-4 s_{3}^{2}\right)}{\left(s_{1}+i s_{2}\right)^{4}}$,

2. $S 2: V=\frac{\alpha}{s_{3}^{2}}+\frac{\beta}{\left(s_{1}+i s_{2}\right)^{2}}+\frac{\gamma\left(s_{1}-i s_{2}\right)}{\left(s_{1}+i s_{2}\right)^{3}}$,

3. $S 4: V=\frac{\alpha}{\left(s_{1}+i s_{2}\right)^{2}}+\frac{\beta s_{3}}{\sqrt{s_{1}^{2}+s_{2}^{2}}}+\frac{\gamma}{\left(s_{1}+i s_{2}\right) \sqrt{s_{1}^{2}+s_{2}^{2}}}$,

4. $S 7: V=\frac{\alpha s_{3}}{\sqrt{s_{1}^{2}+s_{2}^{2}}}+\frac{\beta s_{1}}{s_{2}^{2} \sqrt{s_{1}^{2}+s_{2}^{2}}}+\frac{\gamma}{s_{2}^{2}}$,

5. $S 8: V=\frac{\alpha s_{2}}{\sqrt{s_{1}^{2}+s_{3}^{2}}}+\frac{\beta\left(s_{2}+i s_{1}+s_{3}\right)}{\sqrt{\left(s_{2}+i s_{1}\right)\left(s_{3}+i s_{1}\right)}}+\frac{\gamma\left(s_{2}+i s_{1}-s_{3}\right)}{\sqrt{\left(s_{2}+i s_{1}\right)\left(s_{3}-i s_{1}\right)}}$,

6. $S 9: V=\frac{\alpha}{s_{1}^{2}}+\frac{\beta}{s_{2}^{2}}+\frac{\gamma}{s_{3}^{2}}$.

Darboux 1 systems: $H \Psi=\left(\frac{1}{4 x}\left(\partial_{x}^{2}+\partial_{y}^{2}\right)+V\right) \Psi=E \Psi$.

1. $D 1 A: V=\frac{b_{1}(2 x-2 b+i y)}{x \sqrt{x-b+i y}}+\frac{b_{2}}{x \sqrt{x-b+i y}}+\frac{b_{3}}{x}+b_{4}$,

2. $D 1 B: V=\frac{b_{1}\left(4 x^{2}+y^{2}\right)}{x}+\frac{b_{2}}{x}+\frac{b_{3}}{x y^{2}}+b_{4}$,

3. $D 1 C: V=\frac{b_{1}\left(x^{2}+y^{2}\right)}{x}+\frac{b_{2}}{x}+\frac{b_{3} y}{x}+b_{4}$.

Darboux 2 systems: $H \Psi=\left(\frac{x^{2}}{x^{2}+1}\left(\partial_{x}^{2}+\partial_{y}^{2}\right)+V\right) \Psi=E \Psi$.

1. D2A: $V=\frac{x^{2}}{x^{2}+1}\left(b_{1}\left(x^{2}+4 y^{2}\right)+\frac{b_{2}}{x^{2}}+b_{3} y\right)+b_{4}$,

2. $D 2 B: V=\frac{x^{2}}{x^{2}+1}\left(b_{1}\left(x^{2}+y^{2}\right)+\frac{b_{2}}{x^{2}}+\frac{b_{3}}{y^{2}}\right)+b_{4}$,

3. $D 2 C: V=\frac{x^{2}}{\sqrt{x^{2}+y^{2}}\left(x^{2}+1\right)}\left(b_{1}+\frac{b_{2}}{y+\sqrt{x^{2}+y^{2}}}+\frac{b_{3}}{y-\sqrt{x^{2}+y^{2}}}\right)+b_{4}$.

Darboux 3 systems: $H \Psi=\left(\frac{1}{2} \frac{e^{2 x}}{e^{x}+1}\left(\partial_{x}^{2}+\partial_{y}^{2}\right)+V\right) \Psi=E \Psi$.

1. D3A: $V=\frac{b_{1}}{1+e^{x}}+\frac{b_{2} e^{x}}{\sqrt{1+2 e^{x+i y}}\left(1+e^{x}\right)}+\frac{b_{3} e^{x+i y}}{\sqrt{1+2 e^{x+i y}}\left(1+e^{x}\right)}+b_{4}$, 
2. D3B: $V=\frac{e^{x}}{e^{x}+1}\left(b_{1}+e^{-\frac{x}{2}}\left(b_{2} \cos \frac{y}{2}+b_{3} \sin \frac{y}{2}\right)\right)+b_{4}$,

3. D3C: $V\left(=\frac{e^{x}}{e^{x}+1}\left(b_{1}+e^{x}\left(\frac{b_{2}}{\cos ^{2} \frac{y}{2}}+\frac{b_{3}}{\sin ^{2} \frac{y}{2}}\right)\right)+b_{4}\right.$,

4. D3D: $V=\frac{e^{2 x}}{1+e^{x}}\left(b_{1} e^{-i y}+b_{2} e^{-2 i y}\right)+\frac{b_{3}}{1+e^{x}}+b_{4}$.

Darboux 4 systems: $H \Psi=\left(-\frac{\sin ^{2} 2 x}{2 \cos 2 x+b}\left(\partial_{x}^{2}+\partial_{y}^{2}\right)+V\right) \Psi=E \Psi$.

1. D4(b)A: $V=\frac{\sin ^{2} 2 x}{2 \cos 2 x+b}\left(\frac{b_{1}}{\sinh ^{2} y}+\frac{b_{2}}{\sinh ^{2} 2 y}\right)+\frac{b_{3}}{2 \cos 2 x+b}+b_{4}$,

2. $D 4(b) B: V=\frac{\sin ^{2} 2 x}{2 \cos 2 x+b}\left(\frac{b_{1}}{\sin ^{2} 2 x}+b_{2} e^{4 y}+b_{3} e^{2 y}\right)+b_{4}$,

3. $D 4(b) C: V=\frac{e^{2 y}}{\frac{b+2}{\sin ^{2} x}+\frac{b-2}{\cos ^{2} x}}\left(\frac{b_{1}}{Z+\left(1-e^{2 y}\right) \sqrt{Z}}+\frac{b_{2}}{Z+\left(1+e^{2 y}\right) \sqrt{Z}}+\frac{b_{3} e^{-2 y}}{\cos ^{2} x}\right)+b_{4}$, $Z=\left(1-e^{2 y}\right)^{2}+4 e^{2 y} \cos ^{2} x$.

\section{Generic Koenigs spaces:}

1. $K[1,1,1,1]: H \Psi=\frac{1}{V\left(b_{1}, b_{2}, b_{3}, b_{4}\right)}\left(\partial_{x}^{2}+\partial_{y}^{2}+V\left(a_{1}, a_{2}, a_{3}, a_{4}\right)\right) \Psi=E \Psi$, $V\left(a_{1}, a_{2}, a_{3}, a_{4}\right)=\frac{a_{1}}{x^{2}}+\frac{a_{2}}{y^{2}}+\frac{4 a_{3}}{\left(x^{2}+y^{2}-1\right)^{2}}-\frac{4 a_{4}}{\left(x^{2}+y^{2}+1\right)^{2}}$,

2. $K[2,1,1]: H \Psi=\frac{1}{V\left(b_{1}, b_{2}, b_{3}, b_{4}\right)}\left(\partial_{x}^{2}+\partial_{y}^{2}+V\left(a_{1}, a_{2}, a_{3}, a_{4}\right)\right) \Psi=E \Psi$, $V\left(a_{1}, a_{2}, a_{3}, a_{4}\right)=\frac{a_{1}}{x^{2}}+\frac{a_{2}}{y^{2}}-a_{3}\left(x^{2}+y^{2}\right)+a_{4}$,

3. $K[2,2]: H \Psi=\frac{1}{V\left(b_{1}, b_{2}, b_{3}, b_{4}\right)}\left(\partial_{x}^{2}+\partial_{y}^{2}+V\left(a_{1}, a_{2}, a_{3}, a_{4}\right)\right) \Psi=E \Psi$, $V\left(a_{1}, a_{2}, a_{3}, a_{4}\right)=\frac{a_{1}}{(x+i y)^{2}}+\frac{a_{2}(x-i y)}{(x+i y)^{3}}+a_{3}-a_{4}\left(x^{2}+y^{2}\right)$,

4. $K[3,1]: H \Psi=\frac{1}{V\left(b_{1}, b_{2}, b_{3}, b_{4}\right)}\left(\partial_{x}^{2}+\partial_{y}^{2}+V\left(a_{1}, a_{2}, a_{3}, a_{4}\right)\right) \Psi=E \Psi$, $V\left(a_{1}, a_{2}, a_{3}, a_{4}\right)=a_{1}-a_{2} x+a_{3}\left(4 x^{2}+y^{2}\right)+\frac{a_{4}}{y^{2}}$,

5. $K[4]: H \Psi=\frac{1}{V\left(b_{1}, b_{2}, b_{3}, b_{4}\right)}\left(\partial_{x}^{2}+\partial_{y}^{2}+V\left(a_{1}, a_{2}, a_{3}, a_{4}\right)\right) \Psi=E \Psi$, $V\left(a_{1}, a_{2}, a_{3}, a_{4}\right)=a_{1}-a_{2}(x+i y)+a_{3}\left(3(x+i y)^{2}+2(x-i y)\right)-a_{4}\left(4\left(x^{2}+y^{2}\right)+2(x+i y)^{3}\right)$,

6. $K[0]: H \Psi=\frac{1}{V\left(b_{1}, b_{2}, b_{3}, b_{4}\right)}\left(\partial_{x}^{2}+\partial_{y}^{2}+V\left(a_{1}, a_{2}, a_{3}, a_{4}\right)\right) \Psi=E \Psi$, $V\left(a_{1}, a_{2}, a_{3}, a_{4}\right)=a_{1}-\left(a_{2} x+a_{3} y\right)+a_{4}\left(x^{2}+y^{2}\right)$.

\subsection{Lie algebras and quadratic algebras}

Important for 2 nd order superintegrable systems are the Lie algebras $\mathfrak{e}(2, \mathbb{C})$ and $\mathfrak{o}(3, \mathbb{C})$. These are the $(K=1)$ symmetry Lie algebras of free (zero potential) systems on constant curvature spaces. Every 2nd order symmetry operator on a constant curvature space takes the form $L=K+W(\mathbf{x})$, where $K$ is a 2 nd order element in the enveloping algebra of $\mathfrak{o}(3, \mathbb{C})$ or $\mathfrak{e}(2, \mathbb{C})$. An important example is $S_{9}$ :

$$
H=J_{1}^{2}+J_{2}^{2}+J_{3}^{2}+\frac{a_{1}}{s_{1}^{2}}+\frac{a_{2}}{s_{2}^{2}}+\frac{a_{3}}{s_{3}^{2}},
$$

where $J_{3}=s_{1} \partial_{s_{2}}-s_{2} \partial_{s_{1}}$ and $J_{2}, J_{3}$ are obtained by cyclic permutations of indices. Basis symmetries are

$$
L_{1}=J_{1}^{2}+\frac{a_{3} s_{2}^{2}}{s_{3}^{2}}+\frac{a_{2} s_{3}^{2}}{s_{2}^{2}}, \quad L_{2}=J_{2}^{2}+\frac{a_{1} s_{3}^{2}}{s_{1}^{2}}+\frac{a_{3} s_{1}^{2}}{s_{3}^{2}}, \quad L_{3}=J_{3}^{2}+\frac{a_{2} s_{1}^{2}}{s_{2}^{2}}+\frac{a_{1} s_{2}^{2}}{s_{1}^{2}}
$$


Theorem 1.1. There is a bijection between quadratic algebras generated by 2 nd order elements in the enveloping algebra of $\mathfrak{o}(3, \mathbb{C})$, called free, and 2 nd order nondegenerate superintegrable systems on the complex 2-sphere. Similarly, there is a bijection between quadratic algebras generated by 2 nd order elements in the enveloping algebra of $\mathfrak{e}(2, \mathbb{C})$ and 2 nd order nondegenerate superintegrable systems on the $2 D$ complex flat space.

The proof of this theorem is constructive [21]. Given a free quadratic algebra $\tilde{Q}$ one can compute the potential $V$ and the symmetries of the quadratic algebra $Q$ of the nondegenerate superintegrable system. These systems are closely related to the special functions of mathematical physics and their properties. The special functions arise in two distinct ways: 1) As separable eigenfunctions of the quantum Hamiltonian. Second order superintegrable systems are multiseparable, (with one exception) $[12,13,14,15,16,17]$. 2) As interbasis expansion coefficients relating distinct separable coordinate eigenbases [22, 23, 31, 35]. Most of the classical special functions in the Digital Library of Mathematical Functions, as well as Wilson polynomials, appear in these ways [34].

In [21] it has been shown that all the 2nd order superintegrable systems are obtained by taking coordinate limits of the generic system $S_{9}$ [20], or are obtained from these limits by a Stäckel transform (an invertible structure preserving mapping of superintegrable systems [12, 13, 14, $15,16,17])$. Analogously all quadratic symmetry algebras of these systems are limits of that of $S_{9}$. These coordinate limits induce limit relations between the special functions associated as eigenfunctions of the superintegrable systems. The limits also induce contractions of the associated quadratic algebras, and via the models of the irreducible representations of these algebras, limit relations between the associated special functions. The Askey scheme for orthogonal functions of hypergeometric type is an example of this [25]. For constant curvature systems the required limits are all induced by Inönü-Wigner-type Lie algebra contractions of $\mathfrak{o}(3, \mathbb{C})$ and $\mathfrak{e}(2, \mathbb{C})[9,33,38]$. Inönü-Wigner-type Lie algebra contractions have long been applied to relate separable coordinate systems and their associated special functions, see, e.g., [10, 11] for some more recent examples, but the application to quadratic algebras is due to the authors and their collaborators.

Recall the definition of (natural) Lie algebra contractions: Let $\left(A ;[;]_{A}\right),\left(B ;[;]_{B}\right)$ be two complex Lie algebras. We say that $B$ is a contraction of $A$ if for every $\epsilon \in(0 ; 1]$ there exists a linear invertible map $t_{\epsilon}: B \rightarrow A$ such that for every $X, Y \in B, \lim _{\epsilon \rightarrow 0} t_{\epsilon}^{-1}\left[t_{\epsilon} X, t_{\epsilon} Y\right]_{A}=[X, Y]_{B}$. Thus, as $\epsilon \rightarrow 0$ the 1-parameter family of basis transformations can become nonsingular but the structure constants of the Lie algebra go to a finite limit, necessarily that of another Lie algebra.

The contractions of the symmetry algebras of constant curvature spaces have long since been classified [21]. There are 6 nontrivial contractions of $\mathfrak{e}(2, \mathbb{C})$ and 4 of $\mathfrak{o}(3, \mathbb{C})$. They are each induced by coordinate limits.

Contractions of quadratic algebras: Just as for Lie algebras we can define a contraction of a quadratic algebra in terms of 1-parameter families of basis changes in the algebra: As $\epsilon \rightarrow 0$ the 1-parameter family of basis transformations becomes singular but the structure constants go to a finite limit [21].

Let $H=H^{(0)}+V, S_{1}=S_{1}^{(0)}+W_{1}, S_{2}=S_{2}^{(0)}+W_{2}$ be a superintegrable system on a constant curvature space with quadratic algebra $Q$ and free quadratic algebra $\tilde{Q}$ of $H^{(0)}, S_{1}^{(0)}, S_{2}^{(0)}$. Motivating idea: Lie algebra contractions induce quadratic algebra contractions. For constant curvature spaces we have

Theorem $1.2([21])$. Every Lie algebra contraction of $A=\mathfrak{e}(2, \mathbb{C})$ or $A=\mathfrak{o}(3, \mathbb{C})$ induces a contraction of a free (zero potential) quadratic algebra $\tilde{Q}$ based on $A$, which in turn induces a contraction of the quadratic algebra $Q$ with potential. This is true for both classical and quantum algebras. 
Similarly the coordinate limit associated with each contraction takes $H$ to a new superintegrable system with the contracted quadratic algebra. This relationship between coordinate limits, Lie algebra contractions and quadratic algebra contractions for superintegrable systems on constant curvature spaces breaks down for Darboux and Koenigs spaces. For Darboux spaces the Lie symmetry algebra is only 1-dimensional, and there is no Lie symmetry algebra at all for Koenigs spaces. Furthermore, there is the issue of finding a more systematic way of classifying the 44 distinct Helmholtz superintegrable eigenvalue systems on different manifolds, and their relations. These issues can be clarified by considering the Helmholtz systems as Laplace equations (with potential) on flat space. This point of view was introduced in the paper [18] and applied in [3] to solve important classification problems in the case $n=3$. As announced in [28], the proper object to study is the conformal symmetry algebra $\mathfrak{s o}(4, C)$ of the flat space Laplacian and its contractions. The basic idea is that families of (Stäckel-equivalent) Helmholtz superintegrable systems on a variety of manifolds correspond to a single conformally superintegrable Laplace equation on flat space. We exploit this relation in the case $n=2$, but it generalizes easily to all dimensions $n \geq 2$. The conformal symmetry algebra for Laplace equations with constant potential on flat space is the conformal algebra $\mathfrak{s o}(n+2, \mathbb{C})$. We review these concepts in Section 2 .

In his 1894 thesis [1] Bôcher introduced a limit procedure based on the roots of quadratic forms to find families of $R$-separable solutions of the ordinary (zero potential) flat space Laplace equation in $n$ dimensions. An important feature of his work was the introduction of special projective coordinates in which the action of the conformal group $\mathfrak{s o}(n+2, \mathbb{C})$ on solutions of the Laplace equation can be linearized. For $n=2$ these are tetraspherical coordinates. In Section 4 we describe in detail the Laplace equation mechanism and how it can be applied to systematize the classification of Helmholtz superintegrable systems and their relations via limits. We show that Bôcher's limit procedure can be interpreted as constructing generalized Inönü-Wigner Lie algebra contractions of $\mathfrak{s o}(4, \mathbb{C})$ to itself. We call these Bôcher contractions and show that they induce contractions of the conformal quadratic algebras associated with Laplace superintegrable systems. All of the limits of the Helmholtz systems classified before for $n=2[21]$ are induced by the larger class of Bôcher contractions.

\section{$22 \mathrm{D}$ conformal superintegrability of the 2 nd order}

Systems of Laplace type are of the form

$$
H \Psi \equiv \Delta_{n} \Psi+V \Psi=0 .
$$

Here $\Delta_{n}$ is the Laplace-Beltrami operator on a real or complex conformally flat $n D$ Riemannian or pseudo-Riemannian manifold. We assume that all functions occurring in this paper are locally analytic, real or complex.) A conformal symmetry of this equation is a partial differential operator $S$ in the variables $\mathbf{x}=\left(x_{1}, \ldots, x_{n}\right)$ such that $[S, H] \equiv S H-H S=R_{S} H$ for some differential operator $R_{S}$. A conformal symmetry maps any solution $\Psi$ of (2.1) to another solution. Two conformal symmetries $S, S^{\prime}$ are identified if $S=S^{\prime}+R H$ for some differential operator $R$, since they agree on the solution space of $(2.1)$. (For short we will say that $S=S^{\prime}, \bmod (H)$ and that $S$ is a symmetry if $[S, H]=0, \bmod (H)$.) The system is conformally superintegrable for $n>2$ if there are $2 n-1$ functionally independent conformal symmetries, $S_{1}, \ldots, S_{2 n-1}$ with

$S_{1}=H$. It is second order conformally superintegrable if each symmetry $S_{i}$ can be chosen to be a differential operator of at most second order.

For $n=2$ the definition must be restricted, since for a potential $V=0$ there will be an infinite-dimensional space of conformal symmetries when $n=2$; every analytic function induces such symmetries. We assume $V \neq 0$, possibly a constant.

Every $2 D$ Riemannian manifold is conformally flat, so we can always find a Cartesian-like coordinate system with coordinates $\mathbf{x} \equiv(x, y) \equiv\left(x_{1}, x_{2}\right)$ such that the Laplace equation takes 
the form

$$
\tilde{H}=\frac{1}{\lambda(x, y)}\left(\partial_{x}^{2}+\partial_{y}^{2}\right)+\tilde{V}(\mathbf{x})=0
$$

However, this equation is equivalent to the flat space equation

$$
H \equiv \partial_{x}^{2}+\partial_{y}^{2}+V(\mathbf{x})=0, \quad V(\mathbf{x})=\lambda(\mathbf{x}) \tilde{V}(\mathbf{x}) .
$$

In particular, the conformal symmetries of (2.2) are identical with the conformal symmetries of (2.3). Indeed, denoting by $\Lambda$ the operator of multiplication by the function $\lambda(x, y)$ and using the operator identity $[A, B C]=B[A, C]+[A, B] C$ we have

$$
[S, H]=[S, \Lambda \tilde{H}]=\Lambda[S, \tilde{H}]+[S, \Lambda] \tilde{H}=\Lambda R \tilde{H}+[S, \Lambda] \tilde{H}=\left(\Lambda R \Lambda^{-1}+[S, \Lambda] \Lambda^{-1}\right) H .
$$

Thus without loss of generality we can assume the manifold is flat space with $\lambda \equiv 1$.

Since the Hamiltonians are formally self-adjoint, without loss of generality we can always assume that a 2 nd order conformal symmetry $S$ is formally self-adjoint:

$$
S=\frac{1}{\lambda} \sum_{k, j=1}^{2} \partial_{k} \cdot\left(\lambda a^{k j}(\mathbf{x})\right) \partial_{j}+W(\mathbf{x}) \equiv S_{0}+W, \quad a^{j k}=a^{k j} .
$$

Equating coefficients of the partial derivatives on both sides of

$$
[S, H]=\left(R^{(1)}(\mathbf{x}) \partial_{x}+R^{(2)}(\mathbf{x}) \partial_{y}\right) H,
$$

we can derive the conditions

$$
a_{i}^{i i}=2 a_{j}^{i j}+a_{i}^{j j}, \quad i \neq j, \quad W_{j}=\sum_{s=1}^{2} a^{s j} V_{s}+a_{j}^{j j} V, \quad k, j=1,2 .
$$

Here a subscript $j$ on $a^{\ell m}, V$ or $W$ denotes differentiation with respect to $x_{j}$. The requirement that $\partial_{x} W_{2}=\partial_{y} W_{1}$ leads to the second order (conformal) Bertrand-Darboux partial differential equation for the potential

$$
a^{12}\left(V_{11}-V_{22}\right)+\left(a^{22}-a^{11}\right) V_{12}+\left(a_{1}^{12}+a_{2}^{22}-a_{2}^{11}\right) V_{1}+\left(a_{1}^{22}-a_{1}^{11}-a_{2}^{12}\right) V_{2}+2 a_{12}^{12} V=0 .
$$

The following results are easy modifications of results for 3D conformal superintegrable systems proved in [18]. For a conformally superintegrable system there are 3 functionally independent symmetries, each leading to a Bertrand-Darboux equation. The result is that the potential function $V$ must satisfy a canonical system of equations of the form

$$
\begin{aligned}
& V_{22}=V_{11}+A^{22}(\mathbf{x}) V_{1}+B^{22}(\mathbf{x}) V_{2}+C^{22}(\mathbf{x}) V, \\
& V_{12}=A^{12}(\mathbf{x}) V_{1}+B^{12}(\mathbf{x}) V_{2}+C^{12}(\mathbf{x}) V .
\end{aligned}
$$

If the integrability conditions for this system (2.4) are satisfied identically, the vector space of solutions $V$ is four-dimensional and we say that the potential is nondegenerate. Otherwise the potential is degenerate and the potential involves $\leq 3$ parameters. In this paper we consider only systems with nondegenerate potentials. Since we can always add the trivial conformal symmetry $\rho(\mathbf{x}) H$ to $S$ we could assume that, say $a_{11}=0$.

In general the space of 2 nd order conformal symmetries could be infinite-dimensional. However, the requirement that $H$ have a multiparameter potential reduces the possible symmetries to a finite-dimensional space. Indeed the conformal Bertrand-Darboux conditions for a 2nd 
order symmetry yields the requirement $\partial_{x y}\left(a^{11}-a^{22}\right)=0$. The result is that the pure derivative terms $S_{0}$ belong to the space spanned by symmetrized products of the conformal Killing vectors

$$
\begin{aligned}
& P_{1}=\partial_{x}, \quad P_{2}=\partial_{y}, \quad J=x \partial_{y}-y \partial_{x}, \quad D=x \partial_{x}+y \partial_{y}, \\
& K_{1}=\left(x^{2}-y^{2}\right) \partial_{x}+2 x y \partial_{y}, \quad K_{2}=\left(y^{2}-x^{2}\right) \partial_{y}+2 x y \partial_{x} .
\end{aligned}
$$

and terms $g(\mathbf{x})\left(\partial_{x}^{2}+\partial_{y}^{2}\right)$, where $g$ is an arbitrary function. For a given multiparameter potential only a subspace of these conformal tensors occurs. Note that on the hypersurface $\mathcal{H}=0$ in phase space all symmetries $g(\mathbf{x}) \mathcal{H}$ vanish, so any two symmetries differing by $g(\mathbf{x}) \mathcal{H}$ can be identified.

\subsection{The conformal Stäckel transform}

We review quickly the concept of the Stäckel transform [24] and extend it to conformally superintegrable systems. Suppose we have a second order conformal superintegrable system

$$
H=\frac{1}{\lambda(x, y)}\left(\partial_{x x}+\partial_{y y}\right)+V(x, y)=0, \quad H=H_{0}+V
$$

with $V$ the general solution for this system, and suppose $U(x, y)$ is a particular potential solution, nonzero in an open set. The Stäckel transform induced by $U$ is the system

$$
\tilde{H}=E, \quad \tilde{H}=\frac{1}{\tilde{\lambda}}\left(\partial_{x x}+\partial_{y y}\right)+\tilde{V}, \quad \text { where } \quad \tilde{\lambda}=\lambda U, \quad \tilde{V}=\frac{V}{U} .
$$

In $[18,28]$ we proved

Theorem 2.1. The transformed (Helmholtz) system $\tilde{H}$ is truly superintegrable.

Note that if $H \Psi=0$ then $\tilde{S} \Psi=S \Psi$ and $H(S \Psi)=0$ so $S$ and $\tilde{S}$ agree on the null space of $H$ and they preserve this null space. This result shows that any second order conformal Laplace superintegrable system admitting a nonconstant potential $U$ can be Stäckel transformed to a Helmholtz superintegrable system. This operation is invertible, but the inverse is not a Stäckel transform. By choosing all possible special potentials $U$ associated with the fixed Laplace system (2.6) we generate the equivalence class of all Helmholtz superintegrable systems (2.7) obtainable through this process. As is easy to check, any two Helmholtz superintegrable systems lie in the same equivalence class if and only if they are Stäckel equivalent in the standard sense, see [28, Theorem 4]. All Helmholtz superintegrable systems are related to conformal Laplace systems in this way, so the study of all Helmholtz superintegrability on conformally flat manifolds can be reduced to the study of all conformal Laplace superintegrable systems on flat space.

In $[12,13,14,15,16,17]$ it is demonstrated that for the 3 -parameter Helmholtz system $H^{\prime}$ and the Stäckel transform $\tilde{H}^{\prime}$,

$$
\begin{aligned}
& H^{\prime}=H_{0}+V^{\prime}=H_{0}+U^{(1)} \alpha_{1}+U^{(2)} \alpha_{2}+U^{(3)} \alpha_{3}, \\
& \tilde{H}^{\prime}=\frac{1}{U^{(1)}} H_{0}+\frac{-U^{(1)} E+U^{(2)} \alpha_{2}+U^{(3)} \alpha_{3}}{U^{(1)}},
\end{aligned}
$$

if $H^{\prime} \Psi=E \Psi$ then $\tilde{H}^{\prime} \Psi=-\alpha_{1} \Psi$. The effect of the Stäckel transform is to replace $\alpha_{1}$ by $-E$ and $E$ by $-\alpha_{1}$. Further, a 2nd order symmetry $S$ of $H^{\prime}$ transforms to the 2nd order symmetry $\tilde{S}$ of $\tilde{H}^{\prime}$ such that $S$ and $\tilde{S}$ agree on eigenspaces of $H^{\prime}$.

We know that the symmetry operators of all 2nd order nondegenerate superintegrable systems in $2 \mathrm{D}$ generate a quadratic algebra of the form

$$
\left[R, S_{1}\right]=f^{(1)}\left(S_{1}, S_{2}, \alpha_{1}, \alpha_{2}, \alpha_{3}, H^{\prime}\right), \quad\left[R, S_{2}\right]=f^{(2)}\left(S_{1}, S_{2}, \alpha_{1}, \alpha_{2}, \alpha_{3}, H^{\prime}\right),
$$




$$
R^{2}=f^{(3)}\left(S_{1}, S_{2}, \alpha_{1}, \alpha_{2}, \alpha_{3}, H^{\prime}\right), \quad R \equiv\left[S_{1}, S_{2}\right],
$$

where $\left\{S_{1}, S_{2}, H\right\}$ is a basis for the 2 nd order symmetries and $\alpha_{1}, \alpha_{2}, \alpha_{3}$ are the parameters for the potential $[12,13,14,15,16,17,32]$. We see that the effect of a Stäckel transform generated by the potential function $U^{(1)}$ is to determine a new superintegrable system with structure

$$
\begin{aligned}
& {\left[\tilde{R}, \tilde{S}_{1}\right]=f^{(1)}\left(\tilde{S}_{1}, \tilde{S}_{2},-\tilde{H}^{\prime}, \alpha_{2}, \alpha_{3},-\alpha_{1}\right), \quad\left[\tilde{R}, \tilde{S}_{2}\right]=f^{(2)}\left(\tilde{S}_{1}, \tilde{S}_{2},-\tilde{H}^{\prime}, \alpha_{2}, \alpha_{3},-\alpha_{1}\right),} \\
& \tilde{R}^{2}=f^{(3)}\left(\tilde{S}_{1}, \tilde{S}_{2},-\tilde{H}^{\prime}, \alpha_{2}, \alpha_{3},-\alpha_{1}\right), \quad \tilde{R} \equiv\left[\tilde{S}_{1}, \tilde{S}_{2}\right] .
\end{aligned}
$$

Of course, the switch of $\alpha_{1}$ and $H^{\prime}$ is only for illustration; there is a Stäckel transform that replaces any $\alpha_{j}$ by $-H^{\prime}$ and $H^{\prime}$ by $-\alpha_{j}$.

Formulas (2.8) and (2.9) are just instances of the quadratic algebras of the superintegrable systems belonging to the equivalence class of a single nondegenerate conformally superintegrable

Hamiltonian $\hat{H}=\partial_{x x}+\partial_{y y}+\sum_{j=1}^{4} \alpha_{j} V^{(j)}(x, y)$. Let $\hat{S}_{1}, \hat{S}_{2}, \hat{H}$ be a basis of 2 nd order conformal symmetries of $\hat{H}$. From the above discussion we can conclude the following.

Theorem 2.2. The symmetries of the $2 D$ nondegenerate conformal superintegrable Hamiltonian $\hat{H}$ generate a quadratic algebra

$$
\begin{aligned}
& {\left[\hat{R}, \hat{S}_{1}\right]=f^{(1)}\left(\hat{S}_{1}, \hat{S}_{2}, \alpha_{1}, \alpha_{2}, \alpha_{3}, \alpha_{4}\right), \quad\left[\hat{R}, \hat{S}_{2}\right]=f^{(2)}\left(\hat{S}_{1}, \hat{S}_{2}, \alpha_{1}, \alpha_{2}, \alpha_{3}, \alpha_{4}\right),} \\
& \hat{R}^{2}=f^{(3)}\left(\hat{S}_{1}, \hat{S}_{2}, \alpha_{1}, \alpha_{2}, \alpha_{3}, \alpha_{4}\right),
\end{aligned}
$$

where $\hat{R}=\left[\hat{S}_{1}, \hat{S}_{2}\right]$ and all identities hold $\bmod (\hat{H})$. A conformal Stäckel transform generated by the potential $V^{(j)}(x, y)$ yields a nondegenerate Helmholtz superintegrable Hamiltonian $\tilde{H}$ with quadratic algebra relations identical to (2.10), except that we make the replacements $\hat{S}_{\ell} \rightarrow \tilde{S}_{\ell}$ for $\ell=1,2$ and $\alpha_{j} \rightarrow-\tilde{H}$. These modified relations (2.10) are now true identities, not $\bmod (\hat{H})$.

\section{Tetraspherical coordinates}

The tetraspherical coordinates $\left(x_{1}, \ldots, x_{4}\right)$ satisfy $x_{1}^{2}+x_{2}^{2}+x_{3}^{2}+x_{4}^{2}=0$ (the null cone) and $\sum_{k=1}^{4} x_{k} \partial_{x_{k}}=0$. They are projective coordinates on the null cone and have 3 degrees of freedom. Their principal advantage over flat space Cartesian coordinates is that the action of the conformal algebra $(2.5)$ and of the conformal group $\sim \mathrm{SO}(4, \mathbb{C})$ is linearized in tetraspherical coordinates.

Relation to Cartesian coordinates $(x, y)$ and coordinates on the 2-sphere $\left(s_{1}, s_{2}, s_{3}\right)$ :

$$
\begin{aligned}
& x_{1}=2 X T, \quad x_{2}=2 Y T, \quad x_{3}=X^{2}+Y^{2}-T^{2}, \quad x_{4}=i\left(X^{2}+Y^{2}+T^{2}\right), \\
& x=\frac{X}{T}=-\frac{x_{1}}{x_{3}+i x_{4}}, \quad y=\frac{Y}{T}=-\frac{x_{2}}{x_{3}+i x_{4}}, \quad x=\frac{s_{1}}{1+s_{3}}, \quad y=\frac{s_{2}}{1+s_{3}}, \\
& s_{1}=\frac{2 x}{x^{2}+y^{2}+1}, \quad s_{2}=\frac{2 y}{x^{2}+y^{2}+1}, \quad s_{3}=\frac{1-x^{2}-y^{2}}{x^{2}+y^{2}+1}, \\
& H=\partial_{x x}+\partial_{y y}+\tilde{V}=\left(x_{3}+i x_{4}\right)^{2}\left(\sum_{k=1}^{4} \partial_{x_{k}}^{2}+V\right)=\left(1+s_{3}\right)^{2}\left(\sum_{j=1}^{3} p_{s_{j}}^{2}+V\right), \\
& \tilde{V}=\left(x_{3}+i x_{4}\right)^{2} V, \quad\left(1+s_{3}\right)=-i \frac{\left(x_{3}+i x_{4}\right)}{x_{4}}, \\
& s_{1}=\frac{i x_{1}}{x_{4}}, \quad s_{2}=\frac{i x_{2}}{x_{4}}, \quad s_{3}=\frac{-i x_{3}}{x_{4}} .
\end{aligned}
$$


Relation to flat space and 2-sphere 1st order conformal constants of the motion: We define

$$
L_{j k}=x_{j} \partial_{x_{k}}-x_{k} \partial_{x_{j}}, \quad 1 \leq j, k \leq 4, \quad j \neq k,
$$

where $L_{j k}=-L_{k j}$. The generators for flat space conformal symmetries are related to these via

$$
\begin{aligned}
& P_{1}=\partial_{x}=L_{13}+i L_{14}, \quad P_{2}=\partial_{y}=L_{23}+i L_{24}, \quad D=i L_{34}, \quad J=L_{12}, \\
& K_{j}=L_{j 3}-i L_{j 4}, \quad j=1,2, \quad D=x \partial_{x}+y \partial_{y}, \quad J=x \partial_{y}-y \partial_{x}, \\
& K_{1}=2 x D-\left(x^{2}+y^{2}\right) \partial_{x}, \quad \ldots .
\end{aligned}
$$

The generators for 2-sphere conformal constants of the motion are related to the $L_{j k}$ via

$$
\begin{aligned}
& L_{12}=J_{12}=s_{1} \partial_{s_{2}}-s_{2} \partial_{s_{1}}, \quad L_{13}=J_{13}, \quad L_{23}=J_{23}, \\
& L_{14}=-i \partial_{s_{1}}, \quad L_{24}=-i \partial_{s_{2}}, \quad L_{34}=-i \partial_{s_{3}} .
\end{aligned}
$$

Note that in identifying tetraspherical coordinates we can always permute the parameters 1-4. Also, we can apply an arbitrary $\mathrm{SO}(4, \mathbb{C})$ transformation to the tetraspherical coordinates, so the above relations between Euclidean and tetraspherical coordinates are far from unique.

2nd order conformal symmetries $\sim H$ : The 11-dimensional space of conformal symmetries $\sim H$ has basis

$$
\begin{array}{lll}
L_{12}^{2}-L_{34}^{2}, \quad L_{13}^{2}-L_{24}^{2}, & L_{23}^{2}-L_{14}^{2}, \quad L_{12}^{2}+L_{13}^{2}+L_{23}^{2}, \quad L_{12} L_{34}+L_{23} L_{14}-L_{13} L_{24}, \\
\left\{L_{13}, L_{14}\right\}+\left\{L_{23}, L_{24}\right\}, & \left\{L_{13}, L_{23}\right\}+\left\{L_{14}, L_{24}\right\}, & \left\{L_{12}, L_{13}\right\}+\left\{L_{34}, L_{24}\right\}, \\
\left\{L_{12}, L_{14}\right\}-\left\{L_{34}, L_{23}\right\}, & \left\{L_{12}, L_{23}\right\}-\left\{L_{34}, L_{14}\right\}, & \left\{L_{12}, L_{24}\right\}+\left\{L_{34}, L_{13}\right\} .
\end{array}
$$

All of this becomes much clearer if we make use of the decomposition $\mathfrak{s o}(4, \mathbb{C}) \equiv \mathfrak{s o}(3, \mathbb{C}) \oplus$ $\mathfrak{s o}(3, \mathbb{C})$ and the functional realization of the Lie algebra. Setting

$$
\begin{array}{lcc}
J_{1}=\frac{1}{2}\left(L_{23}-L_{14}\right), & J_{2}=\frac{1}{2}\left(L_{13}+L_{24}\right), & J_{3}=\frac{1}{2}\left(L_{12}-L_{34}\right), \\
K_{1}=\frac{1}{2}\left(L_{23}+L_{14}\right), & K_{2}=\frac{1}{2}\left(L_{13}-L_{24}\right), & K_{3}=\frac{1}{2}\left(L_{12}+L_{34}\right),
\end{array}
$$

we have $\left[J_{i}, J_{j}\right]=\epsilon_{i j k} J_{k},\left[K_{i}, K_{j}\right]=\epsilon_{i j k} K_{k},\left[J_{i}, K_{j}\right]=0$. In the variables $z=x+i y, \bar{z}=x-i y$ :

$$
\begin{aligned}
& J_{1}=\frac{1}{2}\left(i \partial_{z}-i z^{2} \partial_{z}\right), \quad J_{2}=\frac{1}{2}\left(\partial_{z}+z^{2} \partial_{z}\right), \quad J_{3}=i z \partial_{z}, \\
& K_{1}=\frac{1}{2}\left(-i \partial_{\bar{z}}+i \bar{z}^{2} \partial_{\bar{z}}\right), \quad K_{2}=\frac{1}{2}\left(\partial_{\bar{z}}+\bar{z}^{2} \partial_{\bar{z}}\right), \quad K_{3}=-i \bar{z} \partial_{\bar{z}},
\end{aligned}
$$

so the $J_{i}$ operators depend only on $z$ and the $K_{j}$ operators depend only on $\bar{z}$. Also $J_{1}^{2}+J_{2}^{2}+J_{3}^{2} \equiv$ $0, K_{1}^{2}+K_{2}^{2}+K_{3}^{2} \equiv 0$. The space of 2nd order elements in the enveloping algebra is thus 21-dimensional and decomposes as $A_{z} \oplus A_{\bar{z}} \oplus A_{z \bar{z}}$, where $A_{z}$ is 5-dimensional with basis $J_{1}^{2}$, $J_{3}^{2},\left\{J_{1}, J_{2}\right\},\left\{J_{1}, J_{3}\right\},\left\{J_{2}, J_{3}\right\}, A_{\bar{z}}$ is 5 -dimensional with basis $K_{1}^{2}, K_{3}^{2},\left\{K_{1}, K_{2}\right\},\left\{K_{1}, K_{3}\right\}$, $\left\{K_{2}, K_{3}\right\}$, and $A_{z \bar{z}}$ is 9-dimensional with basis $J_{i} K_{j}, 1 \leq i, j \leq 3$. Note that all of the elements of $A_{z \bar{z}}$ are $\sim H$, whereas none of the nonzero elements of $A_{z}, A_{\bar{z}}$ have this property. Here, the transposition $J_{i} \leftrightarrow K_{i}$ is a conformal equivalence.

\subsection{Classification of nondegenerate conformally superintegrable systems}

With this simplification it becomes feasible to classify all conformally 2nd order superintegrable systems with nondegenerate potential. Since every such system has generators $S^{(1)}=$ $S_{0}^{(1)}+W_{1}(z, \bar{z}), S^{(2)}=S_{0}^{(2)}+W_{2}(z, \bar{z})$, it is sufficient to classify, up to $\operatorname{SO}(4, \mathbb{C})$ conjugacy, all free conformal quadratic algebras with generators $S_{0}^{(1)}, S_{0}^{(2)}, \bmod H_{0}\left(H_{0}=\partial_{z \bar{z}}\right)$ and then to determine for which of these free conformal algebras the integrability conditions for equations (2.4) hold identically, so that the system admits a nondegenerate potential $\tilde{V}(z, \bar{z})$ which can be computed. The classification breaks up into the following possible cases: 
- Case 1: $S_{0}^{(1)}, S_{0}^{(2)} \in A_{z}$. (This is conformally equivalent to $S_{0}^{(1)}, S_{0}^{(2)} \in A_{\bar{z}}$.) The possible free conformal quadratic algebras of this type, classified up to $\mathrm{SO}(3, \mathbb{C})$ conjugacy mod $J_{1}^{2}+$ $J_{2}^{2}+J_{3}^{2}$ can easily be obtained from the computations in [21]. They are the pairs
1) $J_{3}^{2}, J_{1}^{2}$,
2) $J_{3}^{2},\left\{J_{1}+i J_{2}, J_{3}\right\}$
3) $J_{3}^{2},\left\{J_{1}, J_{3}\right\}$,
4) $\left\{J_{2}, J_{2}+i J_{1}\right\},\left\{J_{2}, J_{3}\right\}$,
5) $J_{3}^{2},\left(J_{1}+i J_{2}\right)^{2}$,
6) $\left\{J_{1}+i J_{2}, J_{3}\right\},\left(J_{1}+i J_{2}\right)^{2}$.

Checking pairs 1)-5) we find that they do not admit a nonzero potential, so they do not correspond to nondegenerate conformal superintegrable systems. This is in dramatic distinction to the results of [21], where for Helmholtz systems on constant curvature spaces there was a 1-1 relationship between free quadratic algebras and nondegenerate superintegrable systems. Pair 6), does correspond to a superintegrable system, the exceptional case $\tilde{V}=f(z)$, where $f(z)$ is arbitrary. (This system is conformally Stäckel equivalent to the singular Euclidean system $E_{15}$.) Equivalently, the system in $A_{\bar{z}}$ with analogous $K$-operators yields the potential $\tilde{V}=f(\bar{z})$, see (3.7) in Section 3.2.

- Case 2: $S_{0}^{(1)}=S_{J}^{(1)}+S_{K}^{(1)}, S_{0}^{(2)}=S_{J}^{(2)}$, where $S_{J}^{(1)}, S_{J}^{(2)}$ are selected from one of the pairs 1)-6) above and $S_{K}^{(1)}$ is a nonzero element of $A_{\bar{z}}$. Again there is a conformally equivalent case, where the roles of $J_{i}$ and $K_{i}$ are switched. To determine the possibilities for $S_{K}^{(1)}$ we classify the 2 nd order elements in the enveloping algebra of $\mathfrak{s o}(3, \mathbb{C})$ up to $\operatorname{SO}(3, \mathbb{C})$ conjugacy, $\bmod K_{1}^{2}+K_{2}^{2}+K_{3}^{2}$. From the computations in [21] we see easily that there are the following representatives for the equivalence classes:
a) $K_{3}^{2}$,
b) $K_{1}^{2}+a K_{2}^{2}, \quad a \neq 0,1$,
c) $\left(K_{1}+i K_{2}\right)^{2}$,
d) $K_{3}^{2}+\left(K_{1}+i K_{2}\right)^{2}$,
e) $\left\{K_{3}, K_{1}+i K_{2}\right\}$.

For pairs 1), 3), 4), 5) above and all choices $a)-e$ ) we find that the integrability conditions are never satisfied, so there are no corresponding nondegenerate superintegrable systems. For pair 2), however, we find that any choice $a$ ) $-e$ ) leads to the same nondegenerate superintegrable system $[2,2]$, see (3.3) in Section 3.2. While it appears that there are multiple generators for this one system, each set of generators maps to any other set by a conformal Stäckel transformation and a change of variable. For pair 6), we find that any choice $a)-e$ ) leads to the same nondegenerate superintegrable system [4], see (3.5) in Section 3.2. Again each set of generators maps to any other set by a conformal Stäckel transformation and a change of variable.

- Case 3: $S_{0}^{(1)}=S_{J}^{(1)}, S_{0}^{(2)}=S_{J}^{(2)}+S_{K}^{(2)}$, where $S_{J}^{(1)}, S_{J}^{(2)}$ are selected from one of the pairs 1)-6) above and $S_{K}^{(2)}$ is a nonzero element of $A_{\bar{z}}$. Again there is a conformally equivalent case, where the roles of $J_{i}$ and $K_{i}$ are switched. To determine the possibilities for $S_{K}^{(2)}$ we classify the 2 nd order elements in the enveloping algebra of $\mathfrak{s o}(3, \mathbb{C})$ up to $\operatorname{SO}(3, \mathbb{C})$ conjugacy, $\bmod K_{1}^{2}+K_{2}^{2}+K_{3}^{2}$. They are $\left.a\right)-e$ ) above. For pairs 1)-4),6) above and all choices $a)-e$ ) the integrability conditions are never satisfied, so there are no corresponding nondegenerate superintegrable systems. For pair 5), however, we find that any choice $a)-e$ ) leads to the same nondegenerate superintegrable system $[2,2]$, see (3.3) in Section 3.2. Again each set of generators maps to any other set (and to any $[2,2]$ generators in Case 2) by a conformal Stäckel transformation and a change of variable.

- Case 4: $S_{0}^{(1)}=S_{J}^{(1)}, S_{0}^{(2)}=S_{K}^{(2)}$, where $S_{J}^{(1)}$ is selected from one of the representatives $\left.a\right)-e$ ) above and $S_{K}^{(2)}$ is selected from one of the analogous representatives $a$ ) $-e$ ) expressed as $K$-operators. We find that each of the 25 sets of generators leads to the single conformally 
superintegrable system [0], see (3.6) in Section 3.2, and each set of generators maps to any other set by a conformal Stäckel transformation and a change of variable.

- Case 5: $S_{0}^{(1)}=S_{J}^{(1)}+S_{K}^{(1)}, S_{0}^{(2)}=S_{J}^{(2)}+S_{K}^{(2)}$, where $S_{J}^{(1)}, S_{J}^{(2)}$ are selected from one of the pairs 1)-6) above and $S_{K}^{(1)}, S_{K}^{(2)}$ are obtained from $S_{J}^{(1)}, S_{J}^{(2)}$, respectively, by replacing each $J_{i}$ by $K_{i}$. We find the following possibilities:

i) $S_{0}^{(1)}=J_{1}^{2}+K_{1}^{2}, S_{0}^{(2)}=J_{3}^{2}+K_{3}^{2}$. This extends to the system $[1,1,1,1]$, see (3.1) in Section 3.2.

ii) $S_{0}^{(1)}=J_{3}^{2}+K_{3}^{2}, S_{0}^{(2)}=\left\{J_{3}, J_{1}+i J_{2}\right\}+\left\{K_{3}, K_{1}+i K_{2}\right\}$. This extends to the system $[2,1,1]$, see (3.2) in Section 3.2.

iii) $S_{0}^{(1)}=J_{3}^{2}+K_{3}^{2}, S_{0}^{(2)}=\left\{J_{1}, J_{3}\right\}+\left\{K_{1}, K_{3}\right\}$. This extends to the system $[1,1,1,1]$, see (3.1) in Section 3.2, again, equivalent to the generators $i$ ) by a conformal Stäckel transformation and a change of variable.

iv) $S_{0}^{(1)}=\left\{J_{1}, J_{2}+i J_{1}\right\}+\left\{K_{1}, K_{2}+i K_{1}\right\}, S_{0}^{(2)}=\left\{J_{2}, J_{3}\right\}+\left\{K_{2}, K_{3}\right\}$. This does not extend to a conformal superintegrable system.

v) $S_{0}^{(1)}=\left(J_{1}+i J_{2}\right)^{2}+\left(K_{1}+i K_{2}\right)^{2}, S_{0}^{(2)}=J_{3}^{2}+K_{3}^{2}$. This extends to the system $[2,1,1]$, see (3.2) in Section 3.2, again, equivalent to the generators $i i$ ) by a conformal Stäckel transformation and a change of variable.

vi) $S_{0}^{(1)}=\left\{J_{3}, J_{1}+i J_{2}\right\}+\left\{K_{3}, K_{1}+i K_{2}\right\}, S_{0}^{(2)}=\left(J_{1}+i J_{2}\right)^{2}+\left(K_{1}+i K_{2}\right)^{2}$, which extends to the system with potential $[3,1]$, see (3.4) in Section 3.2.

Example 3.1. We describe how apparently distinct superintegrable systems of a fixed type are actually the same. In Case 2 consider the system with generators $\left\{J_{1}+i J_{2}, J_{3}\right\}+\left(K_{1}+\right.$ $\left.i K_{2}\right)^{2},\left(J_{1}+i J_{2}\right)^{2}$. This extends to the conformally superintegrable system [4] with flat space Hamiltonian operator $H_{1}=\partial_{z \bar{z}}+V^{(1)}$, where $V^{(1)}=2 k_{3} z \bar{z}+2 k_{4} z+k_{3} \bar{z}^{3}+3 k_{4} \bar{z}^{2}+k_{1} \bar{z}+k_{2}$. The system with generators $\left\{J_{1}+i J_{2}, J_{3}\right\}+K_{3}^{2}+\left(K_{1}+i K_{2}\right)^{2},\left(J_{1}+i J_{2}\right)^{2}$ again extends to the conformally superintegrable system [4]. Indeed, replacing $z, \bar{z}$ by $Z, \bar{Z}$ to distinguish the two systems, we find the 2nd flat space Hamiltonian operator $H_{2}=\partial_{Z \bar{Z}}+V^{(2)}$, where

$$
V^{(2)}=\frac{c_{3} \operatorname{arcsinh}^{3}(\bar{Z})+3 c_{4} \operatorname{arcsinh}^{2}(\bar{Z})+\left(2 c_{3} Z+c_{1}\right) \operatorname{arcsinh}(\bar{Z})+2 c_{4} Z+c_{2}}{\sqrt{1-\bar{Z}^{2}}} .
$$

Now we perform a conformal Stäckel transform on $H_{2}$ to obtain the new flat space system

$$
\begin{aligned}
\tilde{H}_{2}= & \sqrt{1-\bar{Z}^{2}} \partial_{Z \bar{Z}}+c_{3} \operatorname{arcsinh}^{3}(\bar{Z})+3 c_{4} \operatorname{arcsinh}^{2}(\bar{Z}) \\
& +\left(2 c_{3} Z+c_{1}\right) \operatorname{arcsinh}(\bar{Z})+2 c_{4} Z+c_{2} .
\end{aligned}
$$

Making the change of variable $\bar{Z}=\sinh W$, we find

$$
\tilde{H}_{2}=\partial_{Z W}+c_{3} W^{3}+3 c_{4} W^{2}+\left(2 c_{3} Z+c_{1}\right) W+2 c_{4} Z+c_{2} .
$$

Thus, with the identifications $Z=z, W=\bar{z}, c_{i}=k_{i}$, we see that $H_{1} \equiv \tilde{H}_{2}$.

This completes the classification. The results are summarized in the next section.

\subsection{The 8 Laplace superintegrable systems with nondegenerate potentials}

The systems are all of the form

$$
\left(\sum_{j=1}^{4} \partial_{x_{j}}^{2}+V(\mathbf{x})\right) \Psi=0, \quad \text { or } \quad\left(\partial_{x}^{2}+\partial_{y}^{2}+\tilde{V}\right) \Psi=0
$$


as a flat space system in Cartesian coordinates. The potentials are

$$
\begin{aligned}
& V_{[1,1,1,1]}=\frac{a_{1}}{x_{1}^{2}}+\frac{a_{2}}{x_{2}^{2}}+\frac{a_{3}}{x_{3}^{2}}+\frac{a_{4}}{x_{4}^{2}}, \\
& \tilde{V}_{[1,1,1,1]}=\frac{a_{1}}{x^{2}}+\frac{a_{2}}{y^{2}}+\frac{4 a_{3}}{\left(x^{2}+y^{2}-1\right)^{2}}-\frac{4 a_{4}}{\left(x^{2}+y^{2}+1\right)^{2}}, \\
& V_{[2,1,1]}=\frac{a_{1}}{x_{1}^{2}}+\frac{a_{2}}{x_{2}^{2}}+\frac{a_{3}\left(x_{3}-i x_{4}\right)}{\left(x_{3}+i x_{4}\right)^{3}}+\frac{a_{4}}{\left(x_{3}+i x_{4}\right)^{2}}, \\
& \tilde{V}_{[2,1,1]}=\frac{a_{1}}{x^{2}}+\frac{a_{2}}{y^{2}}-a_{3}\left(x^{2}+y^{2}\right)+a_{4}, \\
& V_{[2,2]}=\frac{a_{1}}{\left(x_{1}+i x_{2}\right)^{2}}+\frac{a_{2}\left(x_{1}-i x_{2}\right)}{\left(x_{1}+i x_{2}\right)^{3}}+\frac{a_{3}}{\left(x_{3}+i x_{4}\right)^{2}}+\frac{a_{4}\left(x_{3}-i x_{4}\right)}{\left(x_{3}+i x_{4}\right)^{3}} \text {, } \\
& \tilde{V}_{[2,2]}=\frac{a_{1}}{(x+i y)^{2}}+\frac{a_{2}(x-i y)}{(x+i y)^{3}}+a_{3}-a_{4}\left(x^{2}+y^{2}\right) \text {, } \\
& V_{[3,1]}=\frac{a_{1}}{\left(x_{3}+i x_{4}\right)^{2}}+\frac{a_{2} x_{1}}{\left(x_{3}+i x_{4}\right)^{3}}+\frac{a_{3}\left(4 x_{1}^{2}+x_{2}^{2}\right)}{\left(x_{3}+i x_{4}\right)^{4}}+\frac{a_{4}}{x_{2}^{2}}, \\
& \tilde{V}_{[3,1]}=a_{1}-a_{2} x+a_{3}\left(4 x^{2}+y^{2}\right)+\frac{a_{4}}{y^{2}} \text {, } \\
& V_{[4]}=\frac{a_{1}}{\left(x_{3}+i x_{4}\right)^{2}}+a_{2} \frac{x_{1}+i x_{2}}{\left(x_{3}+i x_{4}\right)^{3}}+a_{3} \frac{3\left(x_{1}+i x_{2}\right)^{2}-2\left(x_{3}+i x_{4}\right)\left(x_{1}-i x_{2}\right)}{\left(x_{3}+i x_{4}\right)^{4}} \\
& +a_{4} \frac{4\left(x_{3}+i x_{4}\right)\left(x_{3}^{2}+x_{4}^{2}\right)+2\left(x_{1}+i x_{2}\right)^{3}}{\left(x_{3}+i x_{4}\right)^{5}}, \\
& \tilde{V}_{[4]}=a_{1}-a_{2}(x+i y)+a_{3}\left(3(x+i y)^{2}+2(x-i y)\right)-a_{4}\left(4\left(x^{2}+y^{2}\right)+2(x+i y)^{3}\right) \text {, } \\
& V_{[0]}=\frac{a_{1}}{\left(x_{3}+i x_{4}\right)^{2}}+\frac{a_{2} x_{1}+a_{3} x_{2}}{\left(x_{3}+i x_{4}\right)^{3}}+a_{4} \frac{x_{1}^{2}+x_{2}^{2}}{\left(x_{3}+i x_{4}\right)^{4}} \text {, } \\
& \tilde{V}_{[0]}=a_{1}-\left(a_{2} x+a_{3} y\right)+a_{4}\left(x^{2}+y^{2}\right) \text {, } \\
& V_{\mathrm{arb}}=\frac{1}{\left(x_{3}+i x_{4}\right)^{2}} f\left(\frac{-x_{1}-i x_{2}}{x_{3}+i x_{4}}\right) \text {, } \\
& \tilde{V}_{\text {arb }}=f(x+i y), \quad f \text { is arbitrary, } \\
& V(1)=a_{1} \frac{1}{\left(x_{1}+i x_{2}\right)^{2}}+a_{2} \frac{1}{\left(x_{3}+i x_{4}\right)^{2}}+a_{3} \frac{\left(x_{3}+i x_{4}\right)}{\left(x_{1}+i x_{2}\right)^{3}}+a_{4} \frac{\left(x_{3}+i x_{4}\right)^{2}}{\left(x_{1}+i x_{2}\right)^{4}}, \\
& \tilde{V}(1)=\frac{a_{1}}{(x+i y)^{2}}+a_{2}-\frac{a_{3}}{(x+i y)^{3}}+\frac{a_{4}}{(x+i y)^{4}} \quad \text { (a special case of (3.7)), } \\
& V(2)^{\prime}=a_{1} \frac{1}{\left(x_{3}+i x_{4}\right)^{2}}+a_{2} \frac{\left(x_{1}+i x_{2}\right)}{\left(x_{3}+i x_{4}\right)^{3}}+a_{3} \frac{\left(x_{1}+i x_{2}\right)^{2}}{\left(x_{3}+i x_{4}\right)^{4}}+a_{4} \frac{\left(x_{1}+i x_{2}\right)^{3}}{\left(x_{3}+i x_{4}\right)^{5}}, \\
& \tilde{V}(2)^{\prime}=a_{1}+a_{2}(x+i y)+a_{3}(x+i y)^{2}+a_{4}(x+i y)^{3} \quad \text { (a special case of (3.7)). }
\end{aligned}
$$

We note that systems (3.8), (3.9) are not the fundamental Bôcher classes; they are merely special cases of the singular system (3.7). We list them because they, and not the general (3.7), appear as contractions of the fundamental systems.

\subsection{Contractions of conformal superintegrable systems with potential induced by generalized Inönü-Wigner contractions}

The basis symmetries $\mathcal{S}^{(j)}=\mathcal{S}_{0}^{(j)}+W^{(j)}, \mathcal{H}=\mathcal{H}_{0}+V$ of a nondegenerate 2nd order conformally superintegrable system determine a conformal quadratic algebra (2.10), and if the parameters of the potential are set equal to 0 , the free system $\mathcal{S}_{0}^{(j)}, \mathcal{H}_{0}, j=1,2$ also determines a conformal quadratic algebra without parameters, which we call a free conformal quadratic algebra. The 
elements of this free algebra belong to the enveloping algebra of $\mathfrak{s o}(4, \mathbb{C})$ with basis $(2.5)$. Since the system is nondegenerate the integrability conditions for the potential are satisfied identically and the full quadratic algebra can be computed from the free algebra, modulo a choice of basis for the 4-dimensional potential space. Once we choose a basis for $\mathfrak{s o}(4, \mathbb{C})$, its enveloping algebra is uniquely determined by the structure constants. Structure relations in the enveloping algebra are continuous functions of the structure constants, so a contraction of one $\mathfrak{s o}(4, \mathbb{C})$ to itself induces a contraction of the enveloping algebras. Then the free conformal quadratic algebra constructed in the enveloping algebra will contract to another free quadratic algebra. (In [21] essentially the same argument was given in more detail for Helmholtz superintegrable systems on constant curvature spaces.)

In this paper we consider a family of contractions of $\mathfrak{s o}(4, \mathbb{C})$ to itself that we call Bôcher contractions. All these contractions are implemented via coordinate transformations. Suppose we have a conformal nondegenerate superintegrable system with free generators $\mathcal{H}_{0}, \mathcal{S}_{0}^{(1)}, \mathcal{S}_{0}^{(2)}$ that determines the conformal and free conformal quadratic algebras $Q$ and $Q^{(0)}$ and has structure functions $A^{i j}(\mathbf{x}), B^{i j}(\mathbf{x}), C^{i j}(\mathbf{x})$ in Cartesian coordinates $\mathbf{x}=(x, y)$. Further, suppose this system contracts to another nondegenerate system $\mathcal{H}_{0}^{\prime}, \mathcal{S}_{0}^{\prime(1)}, \mathcal{S}_{0}^{(2)}$ with conformal quadratic algebra $Q^{\prime(0)}$. We show here that this contraction induces a contraction of the associated nondegenerate superintegrable system $\mathcal{H}=\mathcal{H}_{0}+V, \mathcal{S}^{(1)}=\mathcal{L}_{0}^{(1)}+W^{(1)}, \mathcal{S}^{(2)}=\mathcal{S}_{0}^{(2)}+W^{(2)}, Q$ to $\mathcal{H}^{\prime}=\mathcal{H}^{\prime}{ }_{0}+V^{\prime}, \mathcal{S}^{(1)}=\mathcal{S}_{0}^{(1)}+W^{(1)^{\prime}}, \mathcal{S}^{(2)}=\mathcal{S}_{0}^{(2)}+W^{(2)^{\prime}}, Q^{\prime}$. The point is that in the contraction process the symmetries $\mathcal{H}^{\prime}{ }_{0}(\epsilon), \mathcal{S}^{\prime(1)}(\epsilon), \mathcal{S}_{0}^{\prime(2)}(\epsilon)$ remain continuous functions of $\epsilon$, linearly independent as quadratic forms, and $\lim _{\epsilon \rightarrow 0} \mathcal{H}_{0}^{\prime}(\epsilon)=\mathcal{H}_{0}^{\prime}, \lim _{\epsilon \rightarrow 0} \mathcal{S}_{0}^{\prime(j)}(\epsilon)=\mathcal{S}_{0}^{\prime(j)}$. Thus the associated functions $A^{i j}(\epsilon), B^{i j}(\epsilon), C^{(i j)}$ will also be continuous functions of $\epsilon$ and $\lim _{\epsilon \rightarrow 0} A^{i j}(\epsilon)=A^{i j}$, $\lim _{\epsilon \rightarrow 0} B^{i j}(\epsilon)=B^{\prime i j}, \lim _{\epsilon \rightarrow 0} C^{i j}(\epsilon)=C^{\prime i j}$. Similarly, the integrability conditions for the potential equations

$$
\begin{aligned}
& V_{22}^{(\epsilon)}=V_{11}^{(\epsilon)}+A^{22}(\epsilon) V_{1}^{(\epsilon)}+B^{22}(\epsilon) V_{2}^{(\epsilon)}+C^{22}(\epsilon) V^{(\epsilon)}, \\
& V_{12}^{(\epsilon)}=A^{12}(\epsilon) V_{1}^{(\epsilon)}+B^{12}(\epsilon) V_{2}^{(\epsilon)}+C^{12}(\epsilon) V^{(\epsilon)}
\end{aligned}
$$

will hold for each $\epsilon$ and in the limit. This means that the 4-dimensional solution space for the potentials $V$ will deform continuously into the 4-dimensional solution space for the potentials $V^{\prime}$. Thus the target space of solutions $V^{\prime}$ (and of the functions $W^{\prime}$ ) is uniquely determined by the free quadratic algebra contraction.

There is an apparent lack of uniqueness in this procedure, since for a nondegenerate superintegrable system one typically chooses a basis $V^{(j)}, j=1, \ldots, 4$ for the potential space and expresses a general potential as $V=\sum_{j=1}^{4} a_{j} V^{(j)}$. Of course the choice of basis for the source system is arbitrary, as is the choice for the target system. Thus the structure equations for the quadratic algebras and the dependence $a_{j}(\epsilon)$ of the contraction constants on $\epsilon$ will vary depending on these choices. However, all such possibilities are related by a basis change matrix.

\subsection{Relation to separation of variables and Bôcher's limit procedures}

Bôcher's analysis $[1,26]$ involves symbols of the form $\left[n_{1}, n_{2}, \ldots, n_{p}\right]$, where $n_{1}+\cdots+n_{p}=4$. These symbols are used to define coordinate surfaces as follows. Consider the quadratic forms

$$
\Omega=x_{1}^{2}+x_{2}^{2}+x_{3}^{2}+x_{4}^{2}=0, \quad \Phi=\frac{x_{1}^{2}}{\lambda-e_{1}}+\frac{x_{2}^{2}}{\lambda-e_{2}}+\frac{x_{3}^{2}}{\lambda-e_{3}}+\frac{x_{4}^{2}}{\lambda-e_{4}}=0 .
$$

If $e_{1}, e_{2}, e_{3}, e_{4}$ are pairwise distinct, the elementary divisors of these two forms are denoted by the symbol $[1,1,1,1]$, see [2]. Given a point in 2D flat space with Cartesian coordinates $\left(x^{0}, y^{0}\right)$, 
there corresponds a set of tetraspherical coordinate $\left(x_{1}^{0}, x_{2}^{0}, x_{3}^{0}, x_{4}^{0}\right)$, unique up to multiplication by a nonzero constant. If we substitute these coordinates into expressions (3.10) we can verify that there are exactly 2 roots $\lambda=\rho, \mu$ such that $\Phi=0$. These are elliptic coordinates. It can be verified that they are orthogonal with respect to the metric $d s^{2}=d x^{2}+d y^{2}$ and that they are $R$-separable for the Laplace equations $\left(\partial_{x}^{2}+\partial_{y}^{2}\right) \Theta=0$ or $\left(\sum_{j=1}^{4} \partial_{x_{j}}^{2}\right) \Theta=0$. Now consider the potential $V_{[1,1,1,1]}=\frac{a_{1}}{x_{1}^{2}}+\frac{a_{2}}{x_{2}^{2}}+\frac{a_{3}}{x_{3}^{2}}+\frac{a_{4}}{x_{4}^{2}}$. It can be verified that this is the only possible potential $V$ such that the Laplace equation $\left(\sum_{j=1}^{4} \partial_{x_{j}}^{2}+V\right) \Theta=0$ is $R$-separable in elliptic coordinates for all choices of the parameters $e_{j}$. The separation is characterized by 2 nd order conformal symmetry operators that are linear in the parameters $e_{j}$. In particular the symmetries span a 3-dimensional subspace of symmetries as the $e_{j}$ are varied, so the system $\left(\sum_{j=1}^{4} \partial_{x_{j}}^{2}+V_{[1,1,1,1]}\right) \Theta=0$ must be conformally superintegrable. We can write this as

$$
H=\left(x_{3}+i x_{4}\right)^{2}\left(\partial_{x_{1}}^{2}+\partial_{x_{2}}^{2}+\partial_{x_{3}}^{2}+\partial_{x_{4}}^{2}+\frac{a_{1}}{x_{1}^{2}}+\frac{a_{2}}{x_{2}^{2}}+\frac{a_{3}}{x_{3}^{2}}+\frac{a_{4}}{x_{4}^{2}}\right),
$$

or in terms of flat space coordinates $x, y$ as

$$
H=\partial_{x}^{2}+\partial_{y}^{2}+\frac{a_{1}}{x^{2}}+\frac{a_{2}}{y^{2}}+\frac{4 a_{3}}{\left(x^{2}+y^{2}-1\right)^{2}}-\frac{4 a_{4}}{\left(x^{2}+y^{2}+1\right)^{2}} .
$$

For the coordinates $s_{i}, i=1,2,3$ we obtain

$$
H=\left(1+s_{3}\right)^{2}\left(\partial_{s_{1}}^{2}+\partial_{s_{2}}^{2}+\partial_{s_{3}}^{2}-\frac{a_{1}}{s_{1}^{2}}-\frac{a_{2}}{s_{2}^{2}}-\frac{a_{3}}{s_{3}^{2}}-a_{4}\right) .
$$

The coordinate curves are described by $[1,1,1, \stackrel{\infty}{1}]$ (because we can always transform to equivalent coordinates for which $e_{4}=\infty$ ) and the corresponding $H \Theta=0$ system is proportional to $S_{9}$, the eigenvalue equation for the generic potential on the 2-sphere, which separates variables in elliptic coordinates $s_{i}^{2}=\frac{\left(\rho-e_{i}\right)\left(\mu-e_{i}\right)}{\left(e_{i}-e_{j}\right)\left(e_{i}-e_{k}\right)}$, where $\left(e_{i}-e_{j}\right)\left(e_{i}-e_{k}\right) \neq 0$ and $i, j, k=1,2,3$. The quantum Hamiltonian when written using these coordinates is equivalent to

$$
\mathcal{H}=\frac{1}{\rho-\mu}\left[P_{\rho}^{2}-P_{\mu}^{2}-\sum_{i=1}^{3} a_{i} \frac{\left(e_{i}-e_{j}\right)\left(e_{i}-e_{k}\right)}{\left(\rho-e_{i}\right)\left(\mu-e_{i}\right)}\right],
$$

where $P_{\lambda}=\sqrt{\prod_{i=1}^{3}\left(\lambda-e_{i}\right)} \partial_{\lambda}$.

\section{$3.5[1,1,1,1]$ to $[2,1,1]$ contraction}

Bôcher provides a recipe to derive separable coordinates in the cases, where some of the $e_{i}$ become equal. In particular, Bôcher shows that the process of making $e_{1} \rightarrow e_{2}$ together with suitable transformations of the $a_{i}^{\prime} s$ produces a conformally equivalent $H$. This corresponds to the choice of coordinate curves obtained by the Bôcher limiting process $[1,1,1,1] \rightarrow[2,1,1]$, i.e., $e_{1}=e_{2}+\epsilon^{2}, x_{1} \rightarrow \frac{i y_{1}}{\epsilon}, x_{2} \rightarrow \frac{y_{1}}{\epsilon}+\epsilon y_{2}, x_{j} \rightarrow y_{j}, j=3,4$, which results in the pair of quadratic forms

$$
\Omega=2 y_{1} y_{2}+y_{3}^{2}+y_{4}^{2}=0, \quad \Phi=\frac{y_{1}^{2}}{\left(\lambda-e_{2}\right)^{2}}+\frac{2 y_{1} y_{2}}{\left(\lambda-e_{2}\right)}+\frac{y_{3}^{2}}{\left(\lambda-e_{3}\right)}+\frac{y_{4}^{2}}{\left(\lambda-e_{4}\right)}=0 .
$$


The coordinate curves with $e_{4}=\infty$ correspond to cyclides with elementary divisors $[2,1, \stackrel{\infty}{1}]$, see [2], i.e.,

$$
\Phi=\frac{y_{1}^{2}}{\left(\lambda-e_{2}\right)^{2}}+\frac{2 y_{1} y_{2}}{\left(\lambda-e_{2}\right)}+\frac{y_{3}^{2}}{\left(\lambda-e_{3}\right)}=0 .
$$

The $\lambda$ roots of $\Phi$ yield planar elliptic coordinates. In order to identify "Cartesian" coordinates on the cone we can choose $y_{1}=\frac{1}{\sqrt{2}}\left(x_{1}^{\prime}+i x_{2}^{\prime}\right), y_{2}=\frac{1}{\sqrt{2}}\left(x_{1}^{\prime}-i x_{2}^{\prime}\right), y_{3}=x_{3}^{\prime}, y_{4}=x_{4}^{\prime}$. Note that the composite linear coordinate mapping

$$
\begin{aligned}
& x_{1}+i x_{2}=\frac{i \sqrt{2}}{\epsilon}\left(x_{1}^{\prime}+i x_{2}^{\prime}\right)+\frac{i \epsilon}{\sqrt{2}}\left(x_{1}^{\prime}-i x_{2}^{\prime}\right), \quad x_{1}-i x_{2}=-\frac{i \epsilon}{\sqrt{2}}\left(x_{1}^{\prime}-i x_{2}^{\prime}\right), \\
& x_{3}=x_{3}^{\prime}, \quad x_{4}=x_{4}^{\prime},
\end{aligned}
$$

satisfies $\lim _{\epsilon \rightarrow 0} \sum_{j=1}^{4} x_{j}^{2}=\sum_{j=1}^{4}{x^{\prime}}_{j}^{2}=0$, preserving the null cone, and it induces a contraction of the Lie algebra $\mathfrak{s o}(4, \mathbb{C})$ to itself. An explicit computation yields the Bôcher contraction $[1,1,1,1] \rightarrow$ $[2,1,1]$ :

$$
\begin{aligned}
& L_{12}^{\prime}=L_{12}, \quad L_{13}^{\prime}=-\frac{i}{\sqrt{2} \epsilon}\left(L_{13}-i L_{23}\right)-\frac{i \epsilon}{\sqrt{2}} L_{13}, \\
& L_{23}^{\prime}=-\frac{i}{\sqrt{2} \epsilon}\left(L_{13}-i L_{23}\right)-\frac{\epsilon}{\sqrt{2}} L_{13}, \quad L_{34}^{\prime}=L_{34}, \\
& L_{14}^{\prime}=-\frac{i}{\sqrt{2} \epsilon}\left(L_{14}-i L_{24}\right)-\frac{i \epsilon}{\sqrt{2}} L_{14}, \quad L_{24}^{\prime}=-\frac{i}{\sqrt{2} \epsilon}\left(L_{14}-i L_{24}\right)-\frac{\epsilon}{\sqrt{2}} L_{14} .
\end{aligned}
$$

Now under the contraction $[1,1,1,1] \rightarrow[2,1,1]$ we have $V_{[1,1,1,1]} \stackrel{\epsilon \rightarrow 0}{\Longrightarrow} V_{[2,1,1]}$, where

$$
\begin{aligned}
& V_{[2,1,1]}=\frac{b_{1}}{\left(x_{1}^{\prime}+i x_{2}^{\prime}\right)^{2}}+\frac{b_{2}\left(x_{1}^{\prime}-i x_{2}^{\prime}\right)}{\left(x_{1}^{\prime}+i x_{2}^{\prime}\right)^{3}}+\frac{b_{3}}{x_{3}^{\prime 2}}+\frac{b_{4}}{x_{4}^{\prime 2}}, \\
& a_{1}=-\frac{1}{2}\left(\frac{b_{1}}{\epsilon^{2}}+\frac{b_{2}}{2 \epsilon^{4}}\right), \quad a_{2}=-\frac{b_{2}}{4 \epsilon^{4}}, \quad a_{3}=b_{3}, \quad a_{4}=b_{4} .
\end{aligned}
$$

Basis of conformal symmetries for original system: Let $H_{0}=\sum_{j=1}^{4} \partial_{x_{j}}^{2}$. A basis is $\left\{H_{0}+V_{[1,1,1,1]}, Q_{12}, Q_{13}\right\}$, where $Q_{j k}=L_{j k}^{2}+a_{j} \frac{x_{k}^{2}}{x_{j}^{2}}+a_{k} \frac{x_{j}^{2}}{x_{k}^{2}}, 1 \leq j<k \leq 4$.

Contraction of basis: Using the notation of (3.12), we have

$$
\begin{aligned}
& H_{0}+V_{[1,1,1,1]} \rightarrow H_{0}^{\prime}+V_{[2,1,1]}, \\
& Q_{12}^{\prime}=Q_{12}-\frac{b_{1}}{2 \epsilon^{2}}-\frac{b_{2}}{2 \epsilon^{4}}=\left(L_{12}^{\prime}\right)^{2}+b_{1}\left(\frac{x_{1}^{\prime}-i x_{2}^{\prime}}{x_{1}^{\prime}+i x_{2}^{\prime}}\right)+b_{2}\left(\frac{x_{1}^{\prime}-i x_{2}^{\prime}}{x_{1}^{\prime}+i x_{2}^{\prime}}\right)^{2}, \\
& Q_{13}^{\prime}=2 \epsilon^{2} Q_{13}=\left(L_{23}^{\prime}-i L_{13}^{\prime}\right)^{2}+\frac{b_{2} x_{3}^{\prime 2}}{\left(x_{1}^{\prime}+i x_{2}^{\prime}\right)^{2}}-\frac{b_{3}\left(x_{1}^{\prime}+i x_{2}^{\prime}\right)^{2}}{x_{3}^{\prime 2}} .
\end{aligned}
$$

If we apply the same $[1,1,1,1] \rightarrow[2,1,1]$ contraction to the $[2,1,1]$ system, the system contracts to itself, but with parameters $c_{1}, \ldots, c_{4}$, where $b_{1}=-\frac{2 c_{1}}{\epsilon^{2}}, b_{2}=\frac{c_{1}}{\epsilon^{2}}+\frac{4 c_{2}}{\epsilon^{4}}, b_{3}=c_{3}, b_{4}=c_{4}$.

If we apply the same contraction to the $[2,2]$ system, the system contracts to itself, but with altered parameters. If we apply the same contraction to the $[3,1]$ system, the system contracts 
to $V(1)$. If we apply the same contraction to the [4] system the system contracts to a system with potential

$$
V[0]=\frac{c_{1}}{\left(x_{1}^{\prime}+i x_{2}^{\prime}\right)^{2}}+\frac{c_{2} x_{3}^{\prime}+c_{3} x_{4}^{\prime}}{\left(x_{1}^{\prime}+i x_{2}^{\prime}\right)^{3}}+c_{4} \frac{x_{3}^{\prime 2}+x_{4}^{\prime 2}}{\left(x_{1}^{\prime}+i x_{2}^{\prime}\right)^{4}}
$$

If we apply this same contraction to the [0], (1) and (2) systems they contract to themselves, but with altered parameters.

The remaining contractions are derived from the Bôcher recipe $[1,26]$.

\section{6 $[1,1,1,1]$ to $[2,2]$ contraction}

$$
\begin{aligned}
& L_{12}^{\prime}=L_{12}, \quad L_{34}^{\prime}=L_{34}, \quad L_{24}^{\prime}+L_{13}^{\prime}=L_{24}+L_{13}, \\
& L_{24}^{\prime}-L_{13}^{\prime}=\left(\epsilon^{2}+\frac{1}{\epsilon^{2}}\right) L_{13}-\frac{1}{\epsilon^{2}}\left(i L_{14}-L_{24}-i L_{23}\right), \\
& L_{23}^{\prime}-L_{14}^{\prime}=2 L_{23}+i L_{13}-i L_{24}, \\
& L_{23}^{\prime}+L_{14}^{\prime}=i\left(\left(\epsilon^{2}-\frac{1}{\epsilon^{2}}\right) L_{13}+\frac{1}{\epsilon^{2}}\left(i L_{14}+L_{24}+i L_{23}\right)\right) .
\end{aligned}
$$

\section{Coordinate implementation:}

$$
\begin{array}{ll}
x_{1}=\frac{i}{\sqrt{2} \epsilon}\left(x_{1}^{\prime}+i x_{2}^{\prime}\right), & x_{2}=\frac{1}{\sqrt{2}}\left(\frac{x_{1}^{\prime}+i x_{2}^{\prime}}{\epsilon}+\epsilon\left(x_{1}^{\prime}-i x_{2}^{\prime}\right)\right), \\
x_{3}=\frac{i}{\sqrt{2} \epsilon}\left(x_{3}^{\prime}+i x_{4}^{\prime}\right), & x_{4}=\frac{1}{\sqrt{2}}\left(\frac{x_{3}^{\prime}+i x_{4}^{\prime}}{\epsilon}+\epsilon\left(x_{3}^{\prime}-i x_{4}^{\prime}\right)\right) .
\end{array}
$$

Limit of 2D potential: $V_{[1,1,1,1]} \stackrel{\epsilon \rightarrow 0}{\Longrightarrow} V_{[2,2]}$, where

$$
V_{[2,2]}=\frac{b_{1}}{\left(x_{1}^{\prime}+i x_{2}^{\prime}\right)^{2}}+\frac{b_{2}\left(x_{1}^{\prime}-i x_{2}^{\prime}\right)}{\left(x_{1}^{\prime}+i x_{2}^{\prime}\right)^{3}}+\frac{b_{3}}{\left(x_{3}^{\prime}+i x_{4}^{\prime}\right)^{2}}+\frac{b_{4}\left(x_{3}^{\prime}-i x_{4}^{\prime}\right)}{\left(x_{3}^{\prime}+i x_{4}^{\prime}\right)^{3}}
$$

and $a_{1}=-\frac{1}{2} \frac{b_{1}}{\epsilon^{2}}-\frac{b_{2}}{4 \epsilon^{4}}, a_{2}=-\frac{b_{2}}{4 \epsilon^{4}}, a_{3}=-\frac{1}{2} \frac{b_{3}}{\epsilon^{2}}-\frac{b_{4}}{4 \epsilon^{4}}, a_{4}=-\frac{b_{4}}{4 \epsilon^{4}}$.

\section{Contracted basis:}

$$
\begin{aligned}
& Q_{12}-\frac{b_{2}}{2 \epsilon^{4}}-\frac{b_{1}}{2 \epsilon^{2}} \rightarrow Q_{1}^{\prime}=L_{12}^{\prime 2}+b_{1} \frac{x_{1}^{\prime}-i x_{2}^{\prime}}{x_{1}^{\prime}+i x_{2}^{\prime}}+b_{2} \frac{\left(x_{1}^{\prime}-i x_{2}^{\prime}\right)^{2}}{\left(x_{1}^{\prime}+i x_{2}^{\prime}\right)^{2}}, \\
& 4 \epsilon^{4} Q_{13} \rightarrow Q_{2}^{\prime}=\left(L_{13}^{\prime}+i L_{14}^{\prime}+i L_{23}^{\prime}-L_{24}^{\prime}\right)^{2}-b_{2} \frac{\left(x_{3}^{\prime}+i x_{4}^{\prime}\right)^{2}}{\left(x_{1}^{\prime}+i x_{2}^{\prime}\right)^{2}}-b_{4} \frac{\left(x_{1}^{\prime}+i x_{2}^{\prime}\right)^{2}}{\left(x_{3}^{\prime}+i x_{4}^{\prime}\right)^{2}} .
\end{aligned}
$$

\section{$3.7[2,1,1]$ to $[3,1]$ contraction}

$$
\begin{aligned}
& L_{24}^{\prime}=\frac{\sqrt{2} i}{2 \epsilon}\left(L_{14}+i L_{24}\right)-L_{34}, L_{14}^{\prime}+i L_{34}^{\prime}=-i \epsilon\left(L_{14}+i L_{24}\right), \\
& L_{14}^{\prime}-i L_{34}^{\prime}=\frac{1}{\epsilon}\left(i L_{14}\left(1+\frac{1}{2 \epsilon^{2}}\right)+L_{24}\left(1-\frac{1}{2 \epsilon^{2}}\right)-\frac{\sqrt{2}}{\epsilon} L_{34}\right), \\
& L_{13}^{\prime}=-L_{12}-2 \sqrt{2} L_{13}\left(\epsilon+2 \epsilon^{3}\right), \quad L_{23}^{\prime}+i L_{12}^{\prime}=4 \epsilon^{3} L_{13}, \\
& L_{23}^{\prime}-i L_{12}^{\prime}=\left(2 \sqrt{2}-\frac{\sqrt{2}}{\epsilon^{2}}\right) L_{12}+\left(8 \epsilon^{3}+4 \epsilon-\frac{2}{\epsilon}+\frac{1}{2 \epsilon^{3}}\right) L_{13}+\frac{i}{2 \epsilon^{3}} L_{23} .
\end{aligned}
$$

\section{Coordinate implementation:}

$$
x_{1}+i x_{2}=-\frac{i \sqrt{2} \epsilon}{2} x_{2}^{\prime}+\frac{\left(i x_{1}^{\prime}-x_{3}^{\prime}\right)}{\epsilon}, \quad x_{1}-i x_{2}=-\epsilon\left(x_{3}^{\prime}+i x_{1}^{\prime}\right)+\frac{3 i \sqrt{2} x_{2}^{\prime}}{4 \epsilon}+\frac{1}{2} \frac{\left(i x_{1}^{\prime}-x_{3}^{\prime}\right)}{\epsilon^{3}},
$$


$x_{3}=-\frac{1}{2} x_{2}^{\prime}-\frac{\sqrt{2}}{2} \frac{\left(x_{1}^{\prime}+i x_{3}^{\prime}\right)}{\epsilon^{2}}, \quad x_{4}=x_{4}^{\prime}$.

Limit of 2D potential: $V_{[2,1,1]} \stackrel{\epsilon \rightarrow 0}{\Longrightarrow} V_{[3,1]}$, where

$$
\begin{aligned}
& V_{[3,1]}=\frac{c_{1}}{\left(x_{1}^{\prime}+i x_{3}^{\prime}\right)^{2}}+\frac{c_{2} x_{2}^{\prime}}{\left(x_{1}^{\prime}+i x_{3}^{\prime}\right)^{3}}+\frac{c_{3}\left(4 x_{2}^{\prime 2}+x_{4}^{\prime 2}\right)}{\left(x_{1}^{\prime}+i x_{3}^{\prime}\right)^{4}}+\frac{c_{4}}{x_{4}^{\prime 2},} \\
& b_{1}=\frac{c_{3}}{\epsilon^{6}}+\frac{\sqrt{2} c_{2}}{4 \epsilon^{4}}-\frac{c_{1}}{\epsilon^{2}}, \quad b_{2}=-\frac{c_{3}}{\epsilon^{4}}-\frac{\sqrt{2} c_{2}}{2 \epsilon^{2}}, \quad b_{3}=\frac{c_{3}}{4 \epsilon^{8}}, \quad b_{4}=c_{4} .
\end{aligned}
$$

Basis of conformal symmetries for original system $H_{0}+V_{[2,1,1]}$ :

$$
\begin{aligned}
& Q_{12}=\left(L_{12}\right)^{2}+b_{1}\left(\frac{x_{1}-i x_{2}}{x_{1}+i x_{2}}\right)+b_{2}\left(\frac{x_{1}-i x_{2}}{x_{1}+i x_{2}}\right)^{2}, \\
& Q_{13}=\left(L_{23}-i L_{13}\right)^{2}+\frac{b_{2} x_{3}^{2}}{\left(x_{1}+i x_{2}\right)^{2}}-\frac{b_{3}\left(x_{1}+i x_{2}\right)^{2}}{x_{3}^{2}} .
\end{aligned}
$$

\section{Contraction of basis:}

$$
\begin{aligned}
H_{0} & +V_{[2,1,1]} \rightarrow H_{0}^{\prime}+V_{[3,1]}, \\
Q_{12}^{\prime} & =-2 \epsilon^{4} Q_{12}+\frac{c_{3}}{2 \epsilon^{4}}-c_{1}=\left(L_{12}^{\prime}-i L_{23}^{\prime}\right)^{2}+\frac{c_{2} x_{2}^{\prime}}{x_{1}^{\prime}+i x_{3}^{\prime}}+\frac{4 c_{3} x_{2}^{2}}{\left(x_{1}^{\prime}+i x_{3}^{\prime}\right)^{2}} \\
Q_{13}^{\prime} & =-\frac{\sqrt{2}}{4}\left(Q_{13}+2 \epsilon^{2} Q_{12}-\frac{3 c_{3}}{2 \epsilon^{6}}-\frac{\sqrt{2} c_{2}}{4 \epsilon^{4}}+c_{1}\right) \\
& =\frac{1}{2}\left\{L_{13}^{\prime}, L_{23}^{\prime}+i L_{12}^{\prime}\right\}+\frac{c_{1} x_{2}^{\prime}}{x_{1}^{\prime}+i x_{3}^{\prime}}+\frac{c_{2}\left(x_{4}^{\prime 2}+4 x_{2}^{\prime 2}\right)}{4\left(x_{1}^{\prime}+i x_{3}^{\prime}\right)^{2}}+\frac{2 c_{3} x_{2}^{\prime}\left(x_{4}^{\prime 2}+2 x_{2}^{\prime 2}\right)}{\left(x_{1}^{\prime}+i x_{3}^{\prime}\right)^{3}} .
\end{aligned}
$$

\section{$3.8[1,1,1,1]$ to $[4]$ contraction}

In this case there is a 2-parameter family of contractions, but all lead to the same result. Let $A, B$ be constants such that $A B(1-A)(1-B)(A-B) \neq 0$.

\section{Coordinate implementation:}

$$
\begin{aligned}
& x_{1}=\frac{i}{\sqrt{2 A B} \epsilon^{3}}\left(x_{1}^{\prime}+i x_{2}^{\prime}\right), \\
& x_{2}=\frac{\left(x_{1}^{\prime}+i x_{2}^{\prime}\right)+\epsilon^{2}\left(x_{3}^{\prime}+i x_{4}^{\prime}\right)+\epsilon^{4}\left(x_{3}^{\prime}-i x_{4}^{\prime}\right)+\epsilon^{6}\left(x_{1}^{\prime}-i x_{2}^{\prime}\right)}{\sqrt{2(A-1)(B-1)} \epsilon^{3}}, \\
& x_{3}=\frac{\left(x_{1}^{\prime}+i x_{2}^{\prime}\right)+A \epsilon^{2}\left(x_{3}^{\prime}+i x_{4}^{\prime}\right)+A^{2} \epsilon^{4}\left(x_{3}^{\prime}-i x_{4}^{\prime}\right)+A^{3} \epsilon^{6}\left(x_{1}^{\prime}-i x_{2}^{\prime}\right)}{\sqrt{2 A(A-1)(A-B)} \epsilon^{3}}, \\
& x_{4}=\frac{\left(x_{1}^{\prime}+i x_{2}^{\prime}\right)+B \epsilon^{2}\left(x_{3}^{\prime}+i x_{4}^{\prime}\right)+B^{2} \epsilon^{4}\left(x_{3}^{\prime}-i x_{4}^{\prime}\right)+B^{3} \epsilon^{6}\left(x_{1}^{\prime}-i x_{2}^{\prime}\right)}{\sqrt{2 B(B-1)(B-A)} \epsilon^{3}}, \\
& i L_{14}^{\prime}+i L_{23}^{\prime}+L_{13}^{\prime}-L_{24}^{\prime}=-2 i \epsilon^{4} \sqrt{A B(A-1)(B-1)} L_{12}, \\
& i L_{14}^{\prime}-i L_{23}^{\prime}-L_{13}^{\prime}-L_{24}^{\prime}=2 i \epsilon^{2}\left(\sqrt{B(A-1)(A-B)} L_{13}-\sqrt{A B(A-1)(B-1)} L_{12}\right), \\
& L_{12}^{\prime}=\frac{\sqrt{B}}{\sqrt{(A-1)(B-1)}} L_{12}+\frac{\sqrt{A}}{\sqrt{(A-1)(A-B)}} L_{13}-\frac{\sqrt{(B-1)(A-B)}}{\sqrt{(A-1)}} L_{14}, \\
& L_{34}^{\prime}=\frac{\sqrt{B(B-1)}}{\sqrt{A(A-1)}} L_{12}-\frac{\sqrt{B(A-B)}}{\sqrt{(A-1)}} L_{13}+i \frac{\sqrt{(B-1)(A-B)}}{\sqrt{A}} L_{23},
\end{aligned}
$$




$$
\begin{gathered}
-i L_{14}^{\prime}+i L_{23}^{\prime}-L_{13}^{\prime}-L_{24}^{\prime}=\frac{2}{\epsilon^{2}}\left(\frac{i(A+B-1)}{\sqrt{A B(A-1)(B-1)}} L_{12}+\frac{i \sqrt{B}}{\sqrt{(A-1)(A-B)}} L_{13}\right. \\
\left.-\frac{\sqrt{A}}{\sqrt{B(B-1)(A-B)}} L_{14}+\frac{\sqrt{(B-1)}}{\sqrt{A(A-B)}} L_{23}-\frac{i \sqrt{(A-1)}}{\sqrt{B(A-B)}} L_{24}\right), \\
i L_{14}^{\prime}+i L_{23}^{\prime}-L_{13}^{\prime}+L_{24}^{\prime}=\frac{2 i}{\epsilon^{4}}\left(-\frac{1}{\sqrt{A B(A-1)(B-1)}}\left(L_{12}+L_{34}\right)\right. \\
\left.+\frac{i}{\sqrt{A(B-1)(A-B)}}\left(L_{14}+L_{23}\right)-\frac{1}{\sqrt{B(A-1)(A-B)}}\left(L_{13}-L_{24}\right)\right) .
\end{gathered}
$$

Limit of 2D potential: $V_{[1,1,1,1]} \stackrel{\epsilon \rightarrow 0}{\Longrightarrow} V_{[4]}$, where

$$
\begin{aligned}
V_{[4]}= & \frac{d_{1}}{\left(x_{1}^{\prime}+i x_{2}^{\prime}\right)^{2}}+\frac{d_{2}\left(x_{3}^{\prime}+i x_{4}^{\prime}\right)}{\left(x_{1}^{\prime}+i x_{2}^{\prime}\right)^{3}}+d_{3}\left(\frac{3\left(x_{3}^{\prime}+i x_{4}^{\prime}\right)^{2}}{\left(x_{1}^{\prime}+i x_{2}^{\prime}\right)^{4}}-2 \frac{\left(x_{1}^{\prime}+i x_{2}^{\prime}\right)\left(x_{3}^{\prime}-i x_{4}^{\prime}\right)}{\left(x_{1}^{\prime}+i x_{2}^{\prime}\right)^{4}}\right) \\
& +d_{4} \frac{4\left(x_{1}^{\prime}+i x_{2}^{\prime}\right)\left(x_{1}^{\prime 2}+x_{2}^{\prime 2}\right)+2\left(x_{3}^{\prime}+i x_{4}^{\prime}\right)^{3}}{\left(x_{1}^{\prime}+i x_{2}^{\prime}\right)^{5}}, \\
a_{1}= & -\frac{d_{4}}{4 A^{2} B^{2} \epsilon^{12}}-\frac{d_{3}}{2 A B^{2} \epsilon^{10}}-\frac{d_{2}}{4 A B \epsilon^{8}}-\frac{d_{1}}{2 A B \epsilon^{6}}, \\
a_{2}= & -\frac{d_{4}}{4(1-A)^{2}(1-B)^{2} \epsilon^{12}}+\frac{d_{2}}{2(1-A)(1-B)^{2} \epsilon^{10}}-\frac{d_{4}}{4(1-A)(1-B) \epsilon^{8}}, \\
a_{3}= & -\frac{d_{4}}{4 A^{2}(1-A)^{2}(A-B)^{2} \epsilon^{12}}, \\
a_{4}= & -\frac{d_{4}}{4 B^{2}(1-B)^{2}(A-B)^{2} \epsilon^{12}}-\frac{d_{3}}{2 B^{2}(1-A)^{2}(A-B) \epsilon^{10}} .
\end{aligned}
$$

In these coordinates a basis for the conformal symmetry algebra is $H, Q_{1}, Q_{2}$, where

$$
\begin{aligned}
Q_{1}= & \frac{1}{4}\left(L_{14}+L_{23}-i L_{13}+i L_{24}\right)^{2}+4 a_{3}\left(\frac{x_{1}+i x_{2}}{x_{3}+i x_{4}}\right)+4 a_{4}\left(\frac{x_{1}+i x_{2}}{x_{3}+i x_{4}}\right)^{2}, \\
Q_{2}= & \frac{1}{2}\left\{L_{23}+L_{14}-i L_{13}+i L_{24}, L_{12}+L_{34}\right\}+\frac{1}{4}\left(L_{14}-L_{23}+i L_{13}+i L_{24}\right)^{2} \\
& +2 a_{1}\left(\frac{x_{1}+i x_{2}}{x_{3}+i x_{4}}\right)+a_{2}\left(2 \frac{x_{1}-i x_{2}}{x_{3}+i x_{4}}-\left(\frac{x_{1}+i x_{2}}{x_{3}+i x_{4}}\right)^{2}\right) \\
& +2 a_{3}\left(6\left(\frac{x_{1}^{2}+x_{2}^{2}}{\left(x_{3}+i x_{4}\right)^{2}}\right)-\left(\frac{x_{1}+i x_{2}}{x_{3}+i x_{4}}\right)^{3}\right) \\
& -4 a_{4}\left(\left(\frac{x_{1}-i x_{2}}{x_{3}+i x_{4}}\right)^{2}-3\left(\frac{\left(x_{1}+i x_{2}\right)^{2}\left(x_{1}-i x_{2}\right)}{\left(x_{3}+i x_{4}\right)^{3}}+\frac{1}{4}\left(\frac{x_{1}+i x_{2}}{x_{3}+i x_{4}}\right)^{4}\right)\right) .
\end{aligned}
$$

Basis of conformal symmetries for original system: $\left\{H_{0}+V_{[1,1,1,1]}, Q_{12}, Q_{13}\right\}$, where $Q_{j k}=\left(x_{j} \partial_{x_{k}}-x_{k} \partial_{x_{j}}\right)^{2}+a_{j} \frac{x_{k}^{2}}{x_{j}^{2}}+a_{k} \frac{x_{j}^{2}}{x_{k}^{2}}, 1 \leq j<k \leq 4$.

Contraction of basis: $H_{0}+V_{[1,1,1,1]} \rightarrow H_{0}^{\prime}+V_{[4]}$,

$$
\begin{aligned}
\epsilon^{8} Q_{12} \sim & \frac{-1}{4(A-1)(B-1) A B}\left(L_{13}^{\prime}-L_{24}^{\prime}+i L_{23}^{\prime}+i L_{14}^{\prime}\right)^{2}+\frac{4 d_{3}\left(x_{3}^{\prime}+i x_{4}^{\prime}\right)}{A B(A-1)(B-1)\left(x_{1}^{\prime}+i x_{2}^{\prime}\right)} \\
& +\frac{d_{4}}{4 A B(A-1)(B-1)}\left[\frac{\left(x_{3}^{\prime}+i x_{4}^{\prime}\right)^{2}}{\left(x_{1}^{\prime}+i x_{2}^{\prime}\right)^{2}}+2 \frac{x_{3}^{\prime}-i x_{4}^{\prime}}{x_{1}^{\prime}+i x_{2}^{\prime}}\right]
\end{aligned}
$$




$$
\begin{aligned}
\epsilon^{5}\left(Q_{12}-\frac{B-A}{(1-B) A} Q_{13}\right) \sim & \frac{-i}{4 A B(B-1)} \\
& \times\left\{L_{13}^{\prime}-L_{24}^{\prime}+i L_{23}^{\prime}+i L_{14}^{\prime}, L_{14}^{\prime}+i L_{13}^{\prime}-L_{23}^{\prime}+i L_{14}^{\prime}\right\} \\
& +\frac{(A+1) d_{1}}{2(B-1) A^{2}}+\frac{d_{2}}{2(B-1) A B} \frac{x_{3}^{\prime}+i x_{4}^{\prime}}{x_{1}^{\prime}+i x_{2}^{\prime}} \\
& +\frac{d_{3}}{2(B-1) A B}\left[3 \frac{\left(x_{3}^{\prime}+i x_{4}^{\prime}\right)^{2}}{\left(x_{1}^{\prime}+i x_{2}^{\prime}\right)^{2}}-2 \frac{x_{3}^{\prime}-i x_{4}^{\prime}}{x_{1}^{\prime}+i x_{2}^{\prime}}\right] \\
& +\frac{d_{4}}{(B-1)(A-1) B}\left[\frac{\left(x_{3}^{\prime}+i x_{4}^{\prime}\right)^{3}}{\left(x_{1}^{\prime}+i x_{2}^{\prime}\right)^{3}}-2 \frac{x_{3}^{\prime 2}+x_{4}^{\prime 2}}{\left(x_{1}^{\prime}+i x_{2}^{\prime}\right)^{2}}\right]
\end{aligned}
$$

The second limit is equivalent to the contracted Hamiltonian, not an independent basis element.

\section{$3.9[2,2]$ to $[4]$ contraction}

$$
\begin{aligned}
L_{12}^{\prime}= & i\left(1+\frac{2}{\epsilon}-\frac{1}{2 \epsilon^{2}}\right) L_{12}+\frac{1}{\epsilon}\left(1-\frac{3}{4 \epsilon}+\frac{1}{4 \epsilon^{2}}\right) L_{13}+\frac{i}{4 \epsilon^{2}}\left(3-\frac{1}{\epsilon}\right) L_{14} \\
& +\frac{i}{4 \epsilon^{2}}\left(3-\frac{1}{\epsilon}\right) L_{23}+\left(3-\epsilon+\frac{3}{4 \epsilon^{2}}-\frac{1}{4 \epsilon^{3}}\right) L_{24}+i\left(\frac{3 \epsilon}{2}-2+\frac{1}{\epsilon}-\frac{1}{2 \epsilon^{2}}\right) L_{34}, \\
L_{12}^{\prime}+ & i L_{24}^{\prime}=\epsilon\left(L_{13}-i L_{14}\right), \quad L_{13}^{\prime}+i L_{34}^{\prime}=\epsilon\left(L_{23}-i L_{24}\right), \\
L_{14}^{\prime}= & (-1+\epsilon) L_{12}+i(1-\epsilon) L_{13}+(1+\epsilon) L_{14}, \quad L_{23}^{\prime}-L_{14}^{\prime}=-L_{14}+L_{23}, \\
L_{13}^{\prime}+ & L_{24}^{\prime}=\left(\frac{1}{2}-\frac{1}{\epsilon}\right) L_{12}+\frac{i}{\epsilon} L_{13}+\frac{1}{2} L_{14}+\frac{1}{2} L_{23}+\left(2+\frac{i}{\epsilon}\right) L_{24}+\left(\epsilon-\frac{1}{2}+\frac{1}{\epsilon}\right) L_{34} .
\end{aligned}
$$

\section{Coordinate implementation:}

$$
\begin{aligned}
& x_{1}=\frac{1}{2}\left(\frac{1}{\epsilon}+\frac{1}{\epsilon^{2}}\right)\left(x_{1}^{\prime}-i x_{4}^{\prime}\right)+\frac{\epsilon}{2}\left(x_{1}^{\prime}+i x_{4}^{\prime}\right)-\left(1+\frac{1}{2 \epsilon}\right)\left(x_{2}^{\prime}-i x_{3}^{\prime}\right)+\frac{1}{2}(\epsilon-1)\left(x_{2}^{\prime}+i x_{3}^{\prime}\right), \\
& x_{2}=\frac{i}{2}\left(\frac{1}{\epsilon}-\frac{1}{\epsilon^{2}}\right)\left(x_{1}^{\prime}-i x_{4}^{\prime}\right)-\frac{i \epsilon}{2}\left(x_{1}^{\prime}+i x_{4}^{\prime}\right)-i\left(1-\frac{1}{2 \epsilon}\right)\left(x_{2}^{\prime}-i x_{3}^{\prime}\right)+\frac{i}{2}(\epsilon+1)\left(x_{2}^{\prime}+i x_{3}^{\prime}\right), \\
& x_{3}=\frac{1}{2}\left(\frac{1}{\epsilon}-\frac{1}{\epsilon^{2}}\right)\left(x_{1}^{\prime}-i x_{4}^{\prime}\right)+\left(-\frac{1}{2}+\frac{1}{\epsilon}\right)\left(x_{2}^{\prime}-i x_{3}^{\prime}\right), \\
& x_{4}=\frac{i}{2}\left(\frac{1}{\epsilon}+\frac{1}{\epsilon^{2}}\right)\left(x_{1}^{\prime}-i x_{4}^{\prime}\right)-i\left(\frac{1}{2}+\frac{1}{\epsilon}\right)\left(x_{2}^{\prime}-i x_{3}^{\prime}\right) .
\end{aligned}
$$

Limit of $2 \mathrm{D}$ potential: $V_{[2,2]} \stackrel{\epsilon \rightarrow 0}{\Longrightarrow} V_{[4]}^{\prime}$,

$$
\begin{aligned}
V_{[4]}^{\prime}= & \frac{e_{1}}{\left(x_{1}^{\prime}-i x_{4}^{\prime}\right)^{2}}+\frac{e_{2}\left(x_{2}^{\prime}-i x_{3}^{\prime}\right)}{\left(x_{1}^{\prime}-i x_{4}^{\prime}\right)^{3}}+e_{3}\left(\frac{3\left(x_{2}^{\prime}-i x_{3}^{\prime}\right)^{2}}{\left(x_{1}^{\prime}-i x_{4}^{\prime}\right)^{4}}+2 \frac{\left(x_{1}^{\prime}-i x_{4}^{\prime}\right)\left(x_{2}^{\prime}+i x_{3}^{\prime}\right)}{\left(x_{1}^{\prime}-i x_{4}^{\prime}\right)^{4}}\right) \\
& +e_{4}\left(\frac{4\left(x_{1}^{\prime}-i x_{4}^{\prime}\right)\left(x_{2}^{\prime 2}+x_{3}^{\prime 2}\right)+2\left(x_{2}^{\prime}-i x_{3}^{\prime}\right)^{3}}{\left(x_{1}^{\prime}-i x_{4}^{\prime}\right)^{5}}\right) \quad(\text { conformally equivalent to } V[4]), \\
b_{1}= & \frac{e_{1}}{\epsilon^{4}}+2 \frac{e_{4}}{\epsilon^{7}}, \quad b_{2}=-\frac{e_{2}}{4 \epsilon^{6}}-\frac{e_{3}}{2 \epsilon^{7}}-\frac{e_{4}}{\epsilon^{8}}, \quad b_{3}=2 \frac{e_{3}}{\epsilon^{6}}-2 \frac{e_{4}}{\epsilon^{7}}, \quad b_{4}=-\frac{e_{2}}{4 \epsilon^{6}}+\frac{3 e_{3}}{2 \epsilon^{7}}-\frac{e_{4}}{\epsilon^{8}}
\end{aligned}
$$

Basis of conformal symmetries for original system: $\left\{H_{0}+V_{[2,2]}, Q_{1}, Q_{3}\right\}$.

Contraction of basis: $H_{0}+V_{[2,2]} \rightarrow H_{0}^{\prime}+V_{[4]}^{\prime}$,

$$
-4 \epsilon^{4}\left(Q_{1}+\frac{k_{4}}{\epsilon^{6}}-\frac{k_{3}}{2 \epsilon^{5}}\right) \rightarrow\left(i L_{13}^{\prime}-L_{12}^{\prime}-i L_{24}^{\prime}-L_{34}^{\prime}\right)^{2}
$$




$$
\begin{aligned}
& \quad+k_{2}+4 k_{3} \frac{x_{2}^{\prime}-i x_{3}^{\prime}}{x_{1}^{\prime}-i x_{4}^{\prime}}-4 k_{4} \frac{\left(x_{2}^{\prime}-i x_{3}^{\prime}\right)^{2}}{\left(x_{1}^{\prime}-i x_{4}^{\prime}\right)^{2}}, \\
& \epsilon^{3}\left(Q_{3}-\frac{2 k_{4}}{\epsilon^{7}}+\frac{k_{3}}{\epsilon^{6}}+\frac{k_{1}}{2 \epsilon^{4}}\right) \rightarrow \frac{i}{2}\left\{L_{23}^{\prime}-L_{14}^{\prime},\left(L_{12}^{\prime}-i L_{13}^{\prime}+L_{24}^{\prime}+L_{34}^{\prime}\right\}\right. \\
& \quad+k_{1} \frac{\left(x_{2}^{\prime}-i x_{3}^{\prime}\right)}{\left(x_{1}^{\prime}-i x_{4}^{\prime}\right)}+k_{2} \frac{\left(x_{2}^{\prime}-i x_{3}^{\prime}\right)^{2}}{\left(x_{1}^{\prime}-i x_{4}^{\prime}\right)^{2}}+k_{3} \frac{3\left(x_{2}^{\prime}-i x_{3}^{\prime}\right)^{3}+2\left(x_{2}^{\prime 2}+x_{3}^{\prime 2}\right)\left(x_{1}^{\prime}-i x_{4}^{\prime}\right)}{\left(x_{1}^{\prime}-i x_{4}^{\prime}\right)^{3}} \\
& \quad-2 k_{4}\left(x_{2}^{\prime}-i x_{3}^{\prime}\right) \frac{\left(x_{2}^{\prime}-i x_{3}^{\prime}\right)^{3}+2\left(x_{2}^{\prime 2}+x_{3}^{\prime 2}\right)\left(x_{1}^{\prime}-i x_{4}^{\prime}\right)}{\left(x_{1}^{\prime}-i x_{4}^{\prime}\right)^{4}} .
\end{aligned}
$$

The second limit is equivalent to the contracted Hamiltonian, not an independent basis element.

\section{$3.10[3,1]$ to $[4]$ contraction}

This contraction is not needed because the $[1,1,1,1] \rightarrow[4]$ contraction takes the $V[3,1]$ to $V[4]$.

\section{$3.11[2,1,1]$ to $[4]$ contraction}

This contraction is not needed because the $[1,1,1,1] \rightarrow[4]$ contraction takes $V[2,1,1]$ to $V[4]$.

\section{$3.12[1,1,1,1]$ to $[3,1]$ contraction}

$$
\begin{aligned}
& -L_{12}^{\prime}+i L_{24}^{\prime}=-a \sqrt{2 a^{2}-2} \epsilon L_{12}, \quad L_{13}^{\prime}=-\frac{i}{\sqrt{a^{2}-1}}\left(L_{13}+a L_{12}\right), \\
& L_{14}^{\prime}+i L_{34}^{\prime}=\sqrt{2} a \epsilon L_{14}, \quad-L_{12}^{\prime}+i L_{23}^{\prime}=i \sqrt{2} a \epsilon L_{23}, \quad L_{24}^{\prime}=i\left(\sqrt{a^{2}-1} L_{24}-i a L_{14}\right), \\
& -L_{14}^{\prime}+i L_{34}^{\prime}=\frac{\sqrt{2}}{\epsilon a \sqrt{a^{2}-1}}\left(L_{34}-\sqrt{a^{2}-1} L_{14}-i a L_{24}\right) .
\end{aligned}
$$

\section{Coordinate implementation:}

$$
\begin{aligned}
& x_{1}=\frac{1}{\sqrt{2} a \epsilon}\left(x_{1}^{\prime}+i x_{3}^{\prime}\right)+\frac{x_{2}^{\prime}}{a}+\frac{a \epsilon}{\sqrt{2}}\left(x_{1}^{\prime}-i x_{3}^{\prime}\right), \quad x_{2}=\frac{i\left(x_{1}^{\prime}+i x_{3}^{\prime}\right)}{\sqrt{2 a^{2}-2} \epsilon}, \\
& x_{3}=-\frac{\left(x_{1}^{\prime}+i x_{3}^{\prime}\right)}{\sqrt{2 a^{2}-2} a \epsilon}+\frac{\sqrt{a^{2}-1}}{a} x_{2}^{\prime}, \quad x_{4}=x_{4}^{\prime}, \quad a(a-1) \neq 0 .
\end{aligned}
$$

Limit of 2D potential: $V_{[1,1,1,1]} \stackrel{\epsilon \rightarrow 0}{\Longrightarrow} V_{[31]}$, where $V[31]$ is given by (3.13) and

$$
\begin{aligned}
& a_{1}=\frac{c_{1}}{2 \epsilon^{2}}+\frac{c_{3}}{4 a^{4} \epsilon^{4}}, \quad a_{2}=\frac{c_{2}}{4 \sqrt{2}\left(a^{2}-1\right)^{2} \epsilon^{3}}+\frac{c_{3}}{4\left(a^{2}-1\right)^{2} \epsilon^{4}}, \\
& a_{3}=\frac{c_{2}}{4 \sqrt{2}\left(a^{2}-1\right)^{2} a^{2} \epsilon^{3}}+\frac{c_{3}}{4\left(a^{2}-1\right)^{2} a^{4} \epsilon^{4}}, \quad a_{4}=c_{4} .
\end{aligned}
$$

Basis of conformal symmetries for original system: $H_{0}+V_{[1,1,1,1]}, Q_{12}, Q_{13}$, where

$$
Q_{j k}=\left(x_{j} \partial_{x_{k}}-x_{k} \partial_{x_{j}}\right)^{2}+a_{j} \frac{x_{k}^{2}}{x_{j}^{2}}+a_{k} \frac{x_{j}^{2}}{x_{k}^{2}}, \quad 1 \leq j<k \leq 4 .
$$

Contracted basis: $H_{0}+V_{[1,1,1,1]} \rightarrow H_{0}^{\prime}+V_{[3,1]}$,

$$
\epsilon^{2}\left(Q_{12}+\frac{c_{3}}{2 a^{2}\left(a^{2}-1\right) \epsilon^{4}}+\frac{\sqrt{2} c_{2}}{a^{2}\left(a^{2}-1\right) \epsilon^{3}}\right) \rightarrow-\frac{c_{1}}{2\left(a^{2}-1\right)}
$$




$$
\begin{gathered}
-\frac{2 c_{3} x_{2}^{\prime 2}}{a^{2}\left(a^{2}-1\right)\left(x_{1}^{\prime}+i x_{3}^{\prime}\right)^{2}}-\frac{c_{2}}{2 a^{2}\left(a^{2}-1\right)\left(x_{1}^{\prime}+i x_{3}^{\prime}\right)}-\frac{1}{2 a^{2}\left(a^{2}-1\right)}\left(L_{12}^{\prime}-i L_{23}^{\prime}\right)^{2}, \\
\epsilon\left(Q_{13}+a^{2} Q_{12}+\frac{\left(a^{2}-1\right) c_{3}}{2 a^{4} \epsilon^{4}}+\frac{\sqrt{2} c_{2}}{8 a^{2} \epsilon^{3}}+\frac{c_{1}\left(a^{2}-1\right)}{2 \epsilon^{2}}\right) \rightarrow \frac{\sqrt{2} c_{1} x_{2}^{\prime}}{x_{1}^{\prime}+i x_{3}^{\prime}} \\
+\frac{\sqrt{2} c_{2}\left(4 x_{2}^{\prime 2}+x_{4}^{\prime 2}\right)}{4\left(x_{1}^{\prime}+i x_{3}^{\prime}\right)^{2}}+\frac{2 \sqrt{2} c_{3} x_{2}^{\prime}\left(2 x_{2}^{\prime 2}+x_{4}^{\prime 2}\right)}{\left(x_{1}^{\prime}+i x_{3}^{\prime}\right)^{3}}+\frac{i \sqrt{2}}{2}\left\{L_{13}^{\prime}, L_{12}^{\prime}-i L_{23}^{\prime}\right\} .
\end{gathered}
$$

\section{$3.13[2,2]$ to $[4]$ contraction}

$$
\begin{aligned}
L_{12}^{\prime}= & i\left(1+\frac{2}{\epsilon}-\frac{1}{2 \epsilon^{2}}\right) L_{12}+\frac{1}{\epsilon}\left(1-\frac{3}{4 \epsilon}+\frac{1}{4 \epsilon^{2}}\right) L_{13}+\frac{i}{4 \epsilon^{2}}\left(3-\frac{1}{\epsilon}\right) L_{14} \\
& +\frac{i}{4 \epsilon^{2}}\left(3-\frac{1}{\epsilon}\right) L_{23}+\left(3-\epsilon+\frac{3}{4 \epsilon^{2}}-\frac{1}{4 \epsilon^{3}}\right) L_{24}+i\left(\frac{3 \epsilon}{2}-2+\frac{1}{\epsilon}-\frac{1}{2 \epsilon^{2}}\right) L_{34}, \\
L_{12}^{\prime}+ & i L_{24}^{\prime}=\epsilon\left(L_{13}-i L_{14}\right), \quad L_{13}^{\prime}+i L_{34}^{\prime}=\epsilon\left(L_{23}-i L_{24}\right), \\
L_{14}^{\prime}= & (-1+\epsilon) L_{12}+i(1-\epsilon) L_{13}+(1+\epsilon) L_{14}, \quad L_{23}^{\prime}-L_{14}^{\prime}=-L_{14}+L_{23}, \\
L_{13}^{\prime}+ & L_{24}^{\prime}=\left(\frac{1}{2}-\frac{1}{\epsilon}\right) L_{12}+\frac{i}{\epsilon} L_{13}+\frac{1}{2} L_{14}+\frac{1}{2} L_{23}+\left(2+\frac{i}{\epsilon}\right) L_{24}+\left(\epsilon-\frac{1}{2}+\frac{1}{\epsilon}\right) L_{34} .
\end{aligned}
$$

\section{Coordinate implementation:}

$$
\begin{aligned}
& x_{1}=\frac{1}{2}\left(\frac{1}{\epsilon}+\frac{1}{\epsilon^{2}}\right)\left(x_{1}^{\prime}-i x_{4}^{\prime}\right)+\frac{\epsilon}{2}\left(x_{1}^{\prime}+i x_{4}^{\prime}\right)-\left(1+\frac{1}{2 \epsilon}\right)\left(x_{2}^{\prime}-i x_{3}^{\prime}\right)+\frac{1}{2}(\epsilon-1)\left(x_{2}^{\prime}+i x_{3}^{\prime}\right), \\
& x_{2}=\frac{i}{2}\left(\frac{1}{\epsilon}-\frac{1}{\epsilon^{2}}\right)\left(x_{1}^{\prime}-i x_{4}^{\prime}\right)-\frac{i \epsilon}{2}\left(x_{1}^{\prime}+i x_{4}^{\prime}\right)-i\left(1-\frac{1}{2 \epsilon}\right)\left(x_{2}^{\prime}-i x_{3}^{\prime}\right)+\frac{i}{2}(\epsilon+1)\left(x_{2}^{\prime}+i x_{3}^{\prime}\right), \\
& x_{3}=\frac{1}{2}\left(\frac{1}{\epsilon}-\frac{1}{\epsilon^{2}}\right)\left(x_{1}^{\prime}-i x_{4}^{\prime}\right)+\left(-\frac{1}{2}+\frac{1}{\epsilon}\right)\left(x_{2}^{\prime}-i x_{3}^{\prime}\right), \\
& x_{4}=\frac{i}{2}\left(\frac{1}{\epsilon}+\frac{1}{\epsilon^{2}}\right)\left(x_{1}^{\prime}-i x_{4}^{\prime}\right)-i\left(\frac{1}{2}+\frac{1}{\epsilon}\right)\left(x_{2}^{\prime}-i x_{3}^{\prime}\right) .
\end{aligned}
$$

Limit of 2D potential: $V_{[2,2]} \stackrel{\epsilon \rightarrow 0}{\Longrightarrow} V_{[4]}^{\prime}$. Conformally equivalent to $V[4]$,

$$
\begin{aligned}
V_{[4]}^{\prime}= & \frac{e_{1}}{\left(x_{1}^{\prime}-i x_{4}^{\prime}\right)^{2}}+\frac{e_{2}\left(x_{2}^{\prime}-i x_{3}^{\prime}\right)}{\left(x_{1}^{\prime}-i x_{4}^{\prime}\right)^{3}}+e_{3}\left(\frac{3\left(x_{2}^{\prime}-i x_{3}^{\prime}\right)^{2}}{\left(x_{1}^{\prime}-i x_{4}^{\prime}\right)^{4}}+2 \frac{\left(x_{1}^{\prime}-i x_{4}^{\prime}\right)\left(x_{2}^{\prime}+i x_{3}^{\prime}\right)}{\left(x_{1}^{\prime}-i x_{4}^{\prime}\right)^{4}}\right) \\
& +e_{4}\left(\frac{4\left(x_{1}^{\prime}-i x_{4}^{\prime}\right)\left(x_{2}^{\prime 2}+x_{3}^{\prime 2}\right)+2\left(x_{2}^{\prime}-i x_{3}^{\prime}\right)^{3}}{\left(x_{1}^{\prime}-i x_{4}^{\prime}\right)^{5}}\right), \\
b_{1}= & \frac{e_{1}}{\epsilon^{4}}+2 \frac{e_{4}}{\epsilon^{7}}, \quad b_{2}=-\frac{e_{2}}{4 \epsilon^{6}}-\frac{e_{3}}{2 \epsilon^{7}}-\frac{e_{4}}{\epsilon^{8}}, \quad b_{3}=2 \frac{e_{3}}{\epsilon^{6}}-2 \frac{e_{4}}{\epsilon^{7}}, \quad b_{4}=-\frac{e_{2}}{4 \epsilon^{6}}+\frac{3 e_{3}}{2 \epsilon^{7}}-\frac{e_{4}}{\epsilon^{8}} .
\end{aligned}
$$

Basis of conformal symmetries for original system: $\left\{H_{0}+V_{[2,2]}, Q_{1}, Q_{3}\right\}$.

Contraction of basis: $H_{0}+V_{[2,2]} \rightarrow H_{0}^{\prime}+V_{[4]}^{\prime}$,

$$
\begin{aligned}
& -4 \epsilon^{4}\left(Q_{1}+\frac{k_{4}}{\epsilon^{6}}-\frac{k_{3}}{2 \epsilon^{5}}\right) \rightarrow\left(i L_{13}^{\prime}-L_{12}^{\prime}-i L_{24}^{\prime}-L_{34}^{\prime}\right)^{2} \\
& \quad+k_{2}+4 k_{3} \frac{x_{2}^{\prime}-i x_{3}^{\prime}}{x_{1}^{\prime}-i x_{4}^{\prime}}-4 k_{4} \frac{\left(x_{2}^{\prime}-i x_{3}^{\prime}\right)^{2}}{\left(x_{1}^{\prime}-i x_{4}^{\prime}\right)^{2}}, \\
& \epsilon^{3}\left(Q_{3}-\frac{2 k_{4}}{\epsilon^{7}}+\frac{k_{3}}{\epsilon^{6}}+\frac{k_{1}}{2 \epsilon^{4}}\right) \rightarrow \frac{i}{2}\left\{L_{23}^{\prime}-L_{14}^{\prime},\left(L_{12}^{\prime}-i L_{13}^{\prime}+L_{24}^{\prime}+L_{34}^{\prime}\right\}\right. \\
& \quad+k_{1} \frac{\left(x_{2}^{\prime}-i x_{3}^{\prime}\right)}{\left(x_{1}^{\prime}-i x_{4}^{\prime}\right)}+k_{2} \frac{\left(x_{2}^{\prime}-i x_{3}^{\prime}\right)^{2}}{\left(x_{1}^{\prime}-i x_{4}^{\prime}\right)^{2}}+k_{3} \frac{3\left(x_{2}^{\prime}-i x_{3}^{\prime}\right)^{3}+2\left(x_{2}^{\prime 2}+x_{3}^{\prime 2}\right)\left(x_{1}^{\prime}-i x_{4}^{\prime}\right)}{\left(x_{1}^{\prime}-i x_{4}^{\prime}\right)^{3}}
\end{aligned}
$$




$$
-2 k_{4}\left(x_{2}^{\prime}-i x_{3}^{\prime}\right) \frac{\left(x_{2}^{\prime}-i x_{3}^{\prime}\right)^{3}+2\left(x_{2}^{\prime 2}+x_{3}^{\prime 2}\right)\left(x_{1}^{\prime}-i x_{4}^{\prime}\right)}{\left(x_{1}^{\prime}-i x_{4}^{\prime}\right)^{4}} .
$$

The second limit is equivalent to the contracted Hamiltonian, not an independent basis element.

\subsection{Summary of Bôcher contractions of Laplace systems}

This is a summary of the results of applying each of the Bôcher contractions to each of the Laplace conformally superintegrable systems. In many cases a single contraction gives rise to rise to more than one result, due to the fact that the indices of the image potential can be permuted and image potential may not be permutation invariant. The details can be found in [27].

1. $[1,1,1,1] \rightarrow[2,1,1]$ contraction:

$$
\begin{aligned}
& V_{[1,1,1,1]} \downarrow V_{[2,1,1]} ; \quad V_{[2,1,1]} \downarrow V_{[2,1,1]}, V_{[2,2]}, V_{[3,1]} ; \quad V_{[2,2]} \downarrow V_{[2,2]}, V_{[0]} ; \\
& V_{[3,1]} \downarrow V_{(1)}, V_{[3,1]} ; \quad V_{[4]} \downarrow V_{[0]}, V_{(2)} ; \quad V_{[0]} \downarrow V_{[0]} ; \quad V_{(1)} \downarrow V_{(1)}, V_{(2)} ; \quad V_{(2)} \downarrow V_{(2)} .
\end{aligned}
$$

2. $[1,1,1,1] \rightarrow[2,2]$ contraction:

$V_{[1,1,1,1]} \downarrow V_{[2,2]} ; \quad V_{[2,1,1]} \downarrow V_{[2,2]}$ (special case of E15); $V_{[2,2]} \downarrow V_{[2,2]}, V_{[0]}$;

$V_{[3,1]} \downarrow V_{(1)}$ (special case of $\left.E_{15}\right) ; \quad V_{[4]} \downarrow V_{(2)} ; \quad V_{[0]} \downarrow V_{[0]} ;$

$V_{(1)} \downarrow V_{(1)}$ (special case of E15); $\quad V_{(2)} \downarrow V_{(2)}$.

3. $[2,1,1] \rightarrow[3,1]$ contraction:

$$
\begin{aligned}
& V_{[1,1,1,1]} \downarrow V_{[3,1]} ; \quad V_{[2,1,1]} \downarrow V_{[3,1]}, V_{[0]} ; \quad V_{[2,2]} \downarrow V_{[0]} ; \quad V_{[3,1]} \downarrow V_{[3,1]}, V_{[0]} ; \quad V_{[4]} \downarrow V_{[0]} ; \\
& V_{[0]} \downarrow V_{[0]} ; \quad V_{(1)} \downarrow V_{(2)} ; \quad V_{(2)} \downarrow V_{(2)} .
\end{aligned}
$$

4. $[1,1,1,1] \rightarrow[4]$ contraction:

$$
\begin{aligned}
& V_{[1,1,1,1]} \downarrow V_{[4]} ; \quad V_{[2,1,1]} \downarrow V_{[4]} ; \quad V_{[2,2]} \downarrow V_{[0]} ; \quad V_{[3,1]} \downarrow V_{[4]} ; \quad V_{[4]} \downarrow V_{[0]}, V_{[4]} ; \\
& V_{[0]} \downarrow V_{[0]} ; \quad V_{(1)} \downarrow V_{(2)} ; \quad V_{(2)} \downarrow V_{(2)} .
\end{aligned}
$$

5. $[2,2] \rightarrow[4]$ contraction:

$$
\begin{aligned}
& V_{[1,1,1,1]} \downarrow V_{[4]} ; \quad V_{[2,1,1]} \downarrow V_{[4]}, V_{(2)} ; \quad V_{[2,2]} \downarrow V_{[4]}, V_{[0]} ; \quad V_{[3,1]} \downarrow V_{(2)} ; \quad V_{[4]} \downarrow V_{(2)} ; \\
& V_{[0]} \downarrow V_{[0]}, V_{(2)} ; \quad V_{(1)} \downarrow V_{(2)} ; \quad V_{(2)} \downarrow V_{(2)} .
\end{aligned}
$$

6. $[1,1,1,1] \rightarrow[3,1]$ contraction:

$$
\begin{array}{llll}
V_{[1,1,1,1]} \downarrow V_{[3,1]} ; & V_{[2,1,1]} \downarrow V_{[3,1]}, V_{[0]} ; & V_{[2,2]} \downarrow V_{[0]} ; & V_{[3,1]} \downarrow V_{[3,1]}, V_{[0]} ; \\
V_{[4]} \downarrow V_{[0]} ; & V_{[0]} \downarrow V_{[0]} ; & V_{(1)} \downarrow V_{(2)} ; & V_{(2)} \downarrow V_{(2)} .
\end{array}
$$

\subsection{Conformal Stäckel transforms of the Laplace systems}

We give the details of the description of the Helmholtz systems that follow from the Laplace system $[1,1,1,1]$ by conformal Stäckel transform

$$
V_{[1,1,1,1]}=\frac{a_{1}}{x_{1}^{2}}+\frac{a_{2}}{x_{2}^{2}}+\frac{a_{3}}{x_{3}^{2}}+\frac{a_{4}}{x_{4}^{2}} .
$$

We write the parameters $a_{j}$ defining the potential $V_{[1,1,1,1]}$ as a vector: $\left(a_{1}, a_{2}, a_{3}, a_{4}\right)$. A Stäckel transform is generated by the potential

$$
U=\frac{b_{1}}{x_{1}^{2}}+\frac{b_{2}}{x_{2}^{2}}+\frac{b_{3}}{x_{3}^{2}}+\frac{b_{4}}{x_{4}^{2}}
$$

corresponding to the vector $\left(b_{1}, b_{2}, b_{3}, b_{4}\right)$. 
1. The potentials $(1,0,0,0)$, and any permutation of the indices $b_{j}$ generate conformal Stäckel transforms to $S 9$.

2. The potentials $(1,1,0,0)$ and $(0,0,1,1)$ generate conformal Stäckel transforms to $S 7$.

3. The potentials $(1,1,1,1),(0,1,0,1),(1,0,1,0),(0,1,1,0)$ and $(1,0,0,1)$ generate conformal Stäckel transforms to $S 8$.

4. The potentials $\left(b_{1}, b_{2}, 0,0\right), b_{1} b_{2} \neq 0, b_{1} \neq b_{2}$, and any permutation of the indices $b_{j}$ generate conformal Stäckel transforms to $D 4 B$.

5. The potentials $(1,1, a, a), a \neq 0,1$, and any permutation of the indices $b_{j}$. generate conformal Stäckel transforms to $D 4 C$.

6. Each potential not proportional to one of these must generate a conformal Stäckel transform to a superintegrable system on a Koenigs space in the family $K[1,1,1,1]$.

Similar details for all of the other Laplace systems are given in [27]. Here, we simply list the Helmholtz systems in each equivalence class.

\subsection{Summary of Stäckel equivalence classes of Helmholtz systems}

1. $[1,1,1,1]: S 9, S 8, S 7, D 4 B, D 4 C, K[1,1,1,1]$.

2. [2,1,1]: $S 4, S 2, E 1, E 16, D 4 A, D 3 B, D 2 B, D 2 C, K[2,1,1]$.

3. [2,2]: $E 8, E 17, E 7, E 19, D 3 C, D 3 D, K[2,2]$.

4. $[3,1]: S 1, E 2, D 1 B, D 2 A, K[3,1]$.

5. [4]: $E 10, E 9, D 1 A, K[4]$.

6. [0]: $E 20, E 11, E 3^{\prime}, D 1 C, D 3 A, K[0]$.

7. (1): special cases of E15.

8. (2): special cases of $E 15$.

\section{Helmholtz contractions from Bôcher contractions}

We describe how Bôcher contractions of conformal superintegrable systems induce contractions of Helmholtz superintegrable systems. The basic idea here is that the procedure of taking a conformal Stäckel transform of a conformal superintegrable system, followed by a Helmholtz contraction yields the same result as taking a Bôcher contraction followed by an ordinary Stäckel transform: The diagrams commute [28]. To describe this process we recall that each of the Bôcher systems classified above can be considered as an equivalence class of Helmholtz superintegrable systems under the Stäckel transform. We now determine the Helmholtz systems in each equivalence class and how they are related.

Consider the conformal Stäckel transforms of the conformal system $[1,1,1,1]$ with potential $V_{[1,1,1,1]}$. The various possibilities are listed in Section 3.15. Let $H$ be the initial Hamiltonian. In terms of tetraspherical coordinates the conformal Stäckel transformed potential will take the form

$$
V=\frac{\frac{a_{1}}{x_{1}^{2}}+\frac{a_{2}}{x_{2}^{2}}+\frac{a_{3}}{x_{3}^{2}}+\frac{a_{4}}{x_{4}^{2}}}{\frac{A_{1}}{x_{1}^{2}}+\frac{A_{2}}{x_{2}^{2}}+\frac{A_{3}}{x_{3}^{2}}+\frac{A_{4}}{x_{4}^{2}}}=\frac{V_{[1,1,1,1]}}{F(\mathbf{x}, \mathbf{A})}, \quad F(\mathbf{x}, \mathbf{A})=\frac{A_{1}}{x_{1}^{2}}+\frac{A_{2}}{x_{2}^{2}}+\frac{A_{3}}{x_{3}^{2}}+\frac{A_{4}}{x_{4}^{2}},
$$

and the transformed Hamiltonian will be $\hat{H}=\frac{1}{F(\mathbf{x}, \mathbf{A})} H$, where the transform is determined by the fixed vector $\left(A_{1}, A_{2}, A_{3}, A_{4}\right)$. Now we apply the Bôcher contraction $[1,1,1,1] \rightarrow[2,1,1]$ to 
this system. In the limit as $\epsilon \rightarrow 0$ the potential $V_{[1,1,1,1]} \rightarrow V_{[2,1,1]},(3.12)$, and $H \rightarrow H^{\prime}$ of the $[2,1,1]$ system. Now consider $F(\mathbf{x}(\epsilon), \mathbf{A})=V^{\prime}\left(\mathbf{x}^{\prime}, A\right) \epsilon^{\alpha}+O\left(\epsilon^{\alpha+1}\right)$, where the integer exponent $\alpha$ depends upon our choice of $\mathbf{A}$. We will provide the theory to show that the system defined by Hamiltonian $\hat{H}^{\prime}=\lim _{\epsilon \rightarrow 0} \epsilon^{\alpha} \hat{H}(\epsilon)=\frac{1}{V^{\prime}\left(\mathbf{x}^{\prime}, A\right)} H^{\prime}$ is a superintegrable system that arises from the system $[2,1,1]$ by a conformal Stäckel transform induced by the potential $V^{\prime}\left(\mathbf{x}^{\prime}, A\right)$. Thus the Helmholtz superintegrable system with potential $V=V_{1,1,1,1} / F$ contracts to the Helmholtz superintegrable system with potential $V_{[2,1,1]} / V^{\prime}$. The contraction is induced by a generalized Inönü-Wigner Lie algebra contraction of the conformal algebra $\mathfrak{s o}(4, \mathbb{C})$. In this case the possibilities for $V^{\prime}$ can be computed easily from the limit expressions (3.11). Then the $V^{\prime}$ can be identified with a $[2,1,1]$ potential from the list in Section 3.2. The results follow. For each $\mathbf{A}$ corresponding to a constant curvature or Darboux superintegrable system $O$ we list the contracted system $O^{\prime}$ and $\alpha$. For Koenigs spaces we will not go into detail but merely give the contraction for a "generic" Koenigs system: One for which there are no rational numbers $r_{j}$, not all 0 , such that $\sum_{j=1}^{4} r_{j} A_{j}=0$. This ensures that the contraction is also "generic". The schematic to keep in mind that relates conformal and regular Stäckel transforms, Bôcher contractions, Helmholtz and Laplace superintegrable systems is Fig. 1.

\section{Schematic of Laplace \& Helmholtz superintegrable systems}

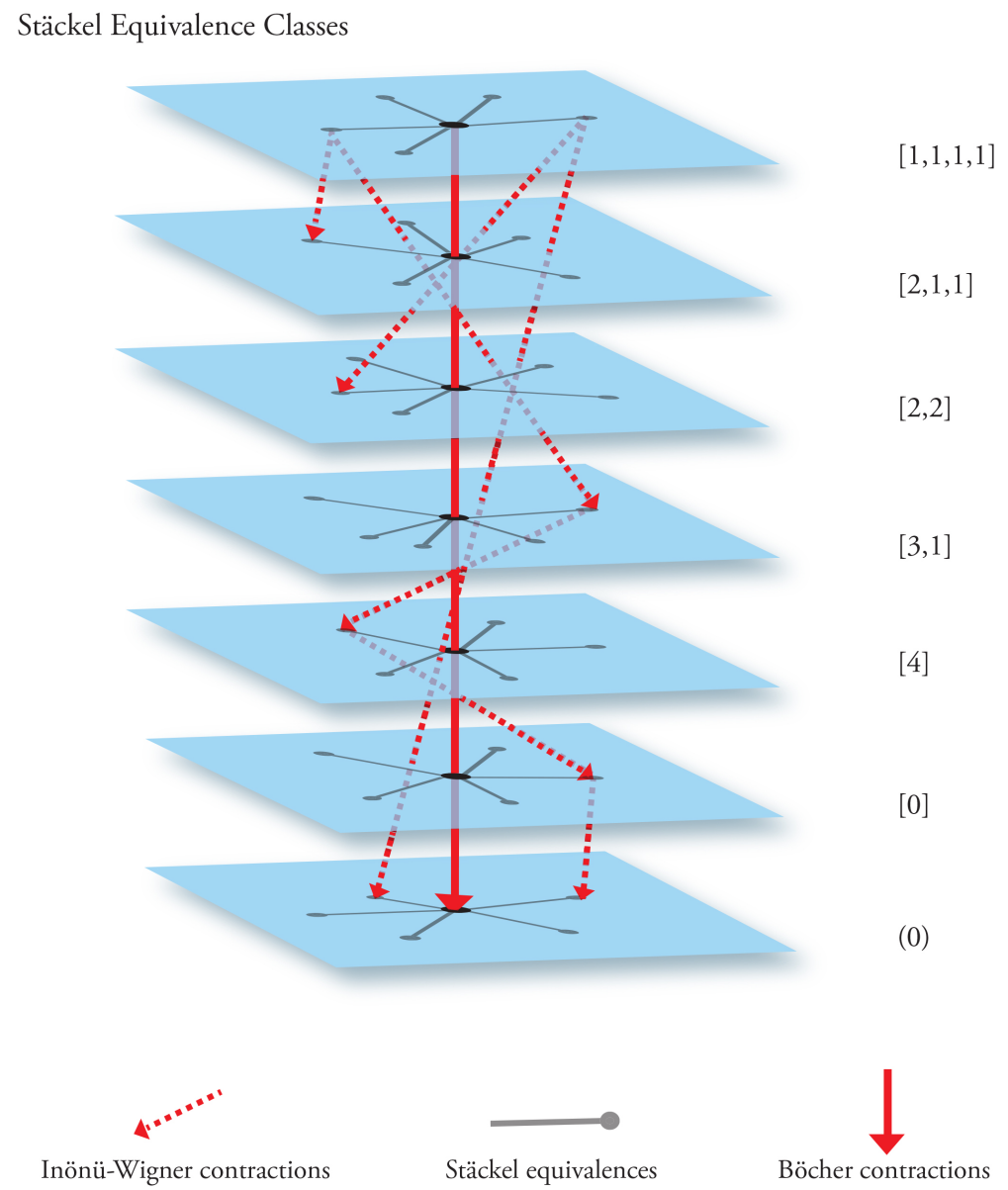

Figure 1. The bigger picture. 
Example 4.1. In Section 3.15, consider Stäckel transform $(1,0,0,0)$, i.e., $U=1 / x_{1}^{2}$. The transformed system is

$$
H=\frac{1}{\frac{1}{x_{1}^{2}}}\left(\sum_{i=1}^{4} \partial_{x_{i}}^{2}\right)+\frac{1}{\frac{1}{x_{1}^{2}}}\left(\frac{a_{1}}{x_{1}^{2}}+\frac{a_{2}}{x_{2}^{2}}+\frac{a_{3}}{x_{3}^{2}}+\frac{a_{4}}{x_{4}^{2}}\right),
$$

which is $S 9$. Now take the $[1,1,1,1] \rightarrow[2,1,1]$ Bôcher contraction, equation (3.12). The sum of the derivatives in $H$ goes to $\sum_{i=1}^{4} \partial_{x_{i}^{\prime}}^{2}$ and the numerator of the potential goes to equation (3.12). However, the denominator $1 / x_{1}^{2}$ goes as $1 / x_{1}^{2}=-2 \epsilon^{2} /\left(\left(x_{1}^{\prime}+i x_{2}^{\prime}\right)^{2}+O\left(\epsilon^{6}\right)\right.$, so $\alpha=2$. Thus, if we set $H^{\prime}=\epsilon^{2} H$ and go to the limit as $\epsilon \rightarrow 0$, we get a contracted system with potential $b_{1}+b_{2}\left(x^{2}+y^{2}\right)+b_{3} / x^{2}+b_{4} / y^{2}$ in Cartesian coordinates, up to a scalar factor -2 . This is $E 1$.

The complicated details of the possible Helmholtz contractions induced by Bôcher contractions of Laplace systems are presented in [27]. Here, we summarize the results. In many cases a single contraction gives rise to more than one result, due to the fact that the indices of the image potential can be permuted and image potential may not be permutation invariant.

\subsection{Summary of Helmholtz contractions}

The superscript for each targeted Helmholtz system is the value of $\alpha$. In each table, corresponding to a single Laplace equation equivalence class, the top line is a list of the Helmholtz systems in the class, and the lower lines are the target systems under the Bôcher contraction.

Table 1. $[1,1,1,1]$ equivalence class contractions.

\begin{tabular}{ccccccc}
\hline contraction & $S_{9}$ & $S_{7}$ & $S_{8}$ & $D_{4} B$ & $D_{4} C$ & $K[1111]$ \\
\hline$[1111] \downarrow[211]$ & $E_{1}^{2}$ & $S_{4}^{0}$ & $S_{4}^{0}$ & $E_{1}^{2}$ & $S_{4}^{0}$ & $D_{4} A^{0}$ \\
& $S_{2}^{0}$ & $S_{2}^{0}$ & $E_{16}^{0}$ & $D_{4} A^{0}$ & $D_{4} A^{0}$ & \\
& & & & $S_{2}^{0}$ & & \\
\hline$[1111] \downarrow[22]$ & $E_{7}^{2}$ & $E_{19}^{4}$ & $E_{17}^{4}$ & $E_{7}^{2}$ & $E_{19}^{1}$ & $E_{7}^{2}$ \\
& & $E_{7}^{2}$ & $E_{19}^{4}$ & & & \\
\hline$[1111] \downarrow[31]$ & $E_{2}^{2}$ & $S_{17}^{2}$ & & & & \\
\hline$[1111] \downarrow[4]$ & $E_{10}^{0}$ & $E_{10}^{6}$ & $E_{10}^{6}$ & $E_{10}^{6}$ & $E_{10}^{6}$ & $E_{10}^{6}$ \\
\hline$[22] \downarrow[4]$ & $E_{10}^{4}$ & $E_{9}^{6}$ & $E_{10}^{5}$ & $E_{10}^{4}$ & $E_{10}^{5}$ & $E_{10}^{4}$ \\
& & $E_{10}^{4}$ & & & & \\
\hline$[211] \downarrow[31]$ & $E_{2}^{6}$ & $E_{9}^{0}$ & & & & \\
& $E_{2}^{4}$ & $E_{2}^{4}$ & $E_{2}^{8}$ & $E_{1}^{6}$ & & $S_{1}^{0}$ \\
& $S_{1}^{0}$ & & & $E_{2}^{4}$ & & \\
\hline
\end{tabular}

\section{Conclusions and discussion}

The use of Lie algebra contractions based on the symmetry groups of constant curvature spaces to construct quadratic algebra contractions of 2nd order 2D Helmholtz superintegrable systems is esthetically pleasing but incomplete, because it doesn't satisfactorily account for Darboux and Koenigs spaces. Also the hierarchy of contractions is confusing. The situation is clarified 
Table 2. $[2,1,1]$ equivalence class contractions.

\begin{tabular}{cccccccccc}
\hline contraction & $S_{4}$ & $S_{2}$ & $E_{1}$ & $E_{16}$ & $D_{4} A$ & $D_{3} B$ & $D_{2} B$ & $D_{2} C$ & $K[211]$ \\
\hline$[111] \downarrow[211]$ & $S_{4}^{0}$ & $S_{2}^{0}$ & $E_{1}^{2}$ & $E_{16}^{4}$ & $D_{4} A^{0}$ & $E_{1}^{2}$ & $S_{2}^{0}$ & $S_{4}^{0}$ & $S_{4}^{0}$ \\
& $E_{17}^{4}$ & $E_{8}^{2}$ & $E_{8}^{0}$ & $E_{17}^{0}$ & $E_{8}^{2}$ & $D_{3} C^{0}$ & $E_{8}^{0}$ & $E_{17}^{0}$ & $D_{3} C^{0}$ \\
& $S_{1}^{0}$ & $S_{1}^{0}$ & $E_{2}^{2}$ & $E_{2}^{2}$ & $S_{1}^{0}$ & $E_{2}^{2}$ & $S_{1}^{0}$ & $S_{1}^{0}$ & $S_{1}^{0}$ \\
& & $E_{2}^{2}$ & & & & $D_{1} B^{3}$ & $E_{2}^{2}$ & & \\
\hline$[1111] \downarrow[22]$ & $E_{17}^{4}$ & $E_{8}^{2}$ & $E_{8}^{2}$ & $E_{17}^{4}$ & $E_{7}^{2}$ & $E_{8}^{2}$ & $E_{7}^{2}$ & $E_{19}^{4}$ & $E_{7}^{2}$ \\
& & & & & $E_{8}^{2}$ & $E_{17}^{2}$ & $E_{8}^{2}$ & $E_{17}^{4}$ & \\
\hline$[1111] \downarrow[31]$ & $S_{1}^{0}$ & $S_{1}^{0}$ & $E_{2}^{2}$ & $E_{2}^{2}$ & $S_{1}^{0}$ & $E_{2}^{2}$ & $E_{1}^{2}$ & $S_{1}^{0}$ & $S_{1}^{0}$ \\
& & & & & & $D_{1} B^{3}$ & & & \\
& $E_{3}^{\prime 2}$ & $E_{3}^{\prime 2}$ & $E_{3}^{\prime 2}$ & $E_{3}^{\prime 2}$ & $E_{3}^{\prime 2}$ & $E_{3}^{\prime 2}$ & $E_{3}^{\prime 2}$ & $E_{3}^{\prime 2}$ & $E_{3}^{\prime 2}$ \\
& & & & & $D_{1} C^{3}$ & $D_{1} C^{3}$ & $D_{1} C^{3}$ & & \\
\hline$[1111] \downarrow[4]$ & $E_{10}^{6}$ & $E_{10}^{6}$ & $E_{10}^{6}$ & $E_{10}^{6}$ & $E_{10}^{6}$ & $E_{10}^{6}$ & $E_{10}^{6}$ & $E_{10}^{6}$ & $E_{10}^{6}$ \\
& & & & & $E_{9}^{8}$ & $E_{9}^{8}$ & $E_{9}^{8}$ & $E_{9}^{8}$ & \\
\hline$[22] \downarrow[4]$ & $E_{10}^{5}$ & $E_{10}^{4}$ & $E_{10}^{4}$ & $E_{10}^{5}$ & $E_{10}^{4}$ & $E_{10}^{4}$ & $E_{10}^{4}$ & $E_{10}^{4}$ & $E_{10}^{4}$ \\
& & & & & & $E_{10}^{5}$ & $E_{10}^{5}$ & & \\
& & & & \multicolumn{2}{c}{ Stäckel transforms of $V(2)$} & & \\
\hline$[211] \downarrow[31]$ & $S_{1}^{0}$ & $S_{1}^{0}$ & $E_{2}^{6}$ & $E_{2}^{8}$ & $S_{1}^{0}$ & $E_{2}^{6}$ & $S_{1}^{0}$ & $S_{1}^{0}$ & $S_{1}^{0}$ \\
& & $E_{2}^{5}$ & & & & & $E_{2}^{5}$ & & \\
& $E_{3}^{\prime \prime}$ & $E_{3}^{\prime 6}$ & $E_{3}^{\prime 4}$ & $E_{3}^{\prime 4}$ & $E_{3}^{\prime 6}$ & $E_{3}^{\prime 6}$ & $E_{3}^{\prime 4}$ & $E_{3}^{\prime 4}$ & $E_{3}^{\prime 4}$ \\
\hline
\end{tabular}

Table 3. $[2,2]$ equivalence class contractions.

\begin{tabular}{cccccccc}
\hline contraction & $E_{8}$ & $E_{17}$ & $E_{7}$ & $E_{19}$ & $D_{3} C$ & $D_{3} D$ & $K[22]$ \\
\hline$[1111] \downarrow[211]$ & $E_{8}^{0}$ & $E_{17}^{0}$ & $E_{7}^{0}$ & $E_{19}^{0}$ & $D_{3} C^{0}$ & $E_{7}^{2}$ & $D_{3} C^{0}$ \\
& $E_{3}^{\prime 2}$ & $E_{3}^{\prime 2}$ & $E_{3}^{\prime 2}$ & $E_{3}^{\prime 2}$ & $E_{3}^{\prime 2}$ & $E_{3}^{\prime 2}$ & $E_{3}^{\prime 2}$ \\
\hline$[1111] \downarrow[22]$ & $E_{8}^{2}$ & $E_{17}^{4}$ & $E_{7}^{2}$ & $E_{19}^{4}$ & $E_{8}^{2}$ & $E_{8}^{2}$ & $E_{7}^{2}$ \\
& $E_{3}^{\prime 2}$ & $E_{11}^{2}$ & $E_{3}^{\prime 2}$ & $E_{11}^{2}$ & $E_{11}^{2}$ & $E_{11}^{2}$ & $E_{11}^{2}$ \\
\hline$[1111] \downarrow[31]$ & $E_{3}^{\prime 2}$ & $E_{3}^{\prime 2}$ & $E_{3}^{\prime 2}$ & $E_{3}^{\prime 2}$ & $E_{3}^{\prime 2}$ & $E_{3}^{\prime 2}$ & $E_{3}^{\prime 2}$ \\
& & & & $E_{11}^{4}$ & $D_{1} C^{3}$ & $D_{1} C^{3}$ & \\
\hline$[1111] \downarrow[4]$ & $E_{3}^{\prime 6}$ & $E_{3}^{\prime 6}$ & $E_{3}^{\prime 6}$ & $E^{\prime 6}$ & $E^{\prime 6}$ & $E_{3}^{\prime 6}$ & $E_{3}^{\prime 6}$ \\
& & & $E_{11}^{8}$ & $E_{11}^{8}$ & $E_{11}^{8}$ & $E_{11}^{8}$ & \\
\hline$[22] \downarrow[4]$ & $E_{10}^{4}$ & $E_{10}^{5}$ & $E_{10}^{4}$ & $E_{10}^{5}$ & $E_{10}^{4}$ & $E_{10}^{4}$ & $E_{10}^{4}$ \\
& & & $E_{9}^{5}$ & $E_{9}^{6}$ & & & \\
& $E_{3}^{\prime 2}$ & $E_{11}^{1}$ & $E_{3}^{\prime 2}$ & $E_{11}^{1}$ & $E_{11}^{1}$ & $E_{11}^{1}$ & $E_{11}^{1}$ \\
& & & $E_{11}^{3}$ & $E_{20}^{4}$ & & & \\
\hline$[211] \downarrow[31]$ & $E_{3}^{\prime 4}$ & $E_{3}^{\prime 4}$ & $E_{3}^{\prime 2}$ & $E_{3}^{\prime 2}$ & $E_{3}^{\prime 4}$ & $D_{1} C^{2}$ & $D_{1} C^{2}$ \\
& $E_{3}^{\prime 6}$ & $E_{3}^{\prime 6}$ & $E_{3}^{\prime 6}$ & $E_{20}{ }^{4}$ & $E_{3}^{\prime 6}$ & $E_{3}^{\prime 6}$ & $E_{3}^{\prime 6}$ \\
& & & & & $D_{1} C^{9}$ & & \\
\hline
\end{tabular}

when one extends these systems to 2nd order Laplace conformally superintegrable systems with conformal symmetry algebra. Classes of Stäckel equivalent Helmholtz superintegrable systems are now recognized as corresponding to a single Laplace superintegrable system on flat space with underlying conformal symmetry algebra $\mathfrak{s o}(4, \mathbb{C})$. The conformal Lie algebra contractions are induced by Bôcher limits of $\mathfrak{s o}(4, \mathbb{C})$ to itself associated with invariants of quadratic forms. 
Table 4. $[3,1]$ equivalence class contractions.

\begin{tabular}{|c|c|c|c|c|c|}
\hline contraction & $S_{1}$ & $E_{2}$ & $D_{1} B$ & $D_{2} A$ & $K[31]$ \\
\hline \multirow[t]{2}{*}[1111]{$\downarrow[211]$} & \multicolumn{5}{|c|}{ Stäckel transforms of $V(1)$} \\
\hline & $S_{1}^{0}$ & $E_{2}^{2}$ & $\begin{array}{c}E_{2}^{2} \\
D_{1} B^{3}\end{array}$ & $\begin{array}{r}E_{2}^{2} \\
D_{2} A\end{array}$ & $S_{1}^{0}$ \\
\hline$[1111] \downarrow[22]$ & \multicolumn{5}{|c|}{ Stäckel transforms of $V(1)$} \\
\hline \multirow[t]{2}{*}[1111]{$\downarrow[31]$} & $S_{1}^{0}$ & & $\begin{array}{c}E_{2}^{2} \\
D_{1} B^{3}\end{array}$ & $E_{2}^{2}$ & $S_{1}^{0}$ \\
\hline & $E_{3}^{\prime 2}$ & $E_{3}^{\prime 2}$ & $\begin{array}{c}E_{3}^{\prime 2} \\
D_{1} C^{3}\end{array}$ & $E_{3}^{\prime 2}$ & $E_{3}^{\prime 2}$ \\
\hline$[1111] \downarrow[4]$ & $E_{10}^{6}$ & $E_{10}^{6}$ & $\begin{array}{c}E_{10}^{6} \\
E_{9}^{8}\end{array}$ & $E_{10}^{6}$ & $E_{10}^{6}$ \\
\hline$[22] \downarrow[4]$ & \multicolumn{5}{|c|}{ Stäckel transforms of $V(2)$} \\
\hline$[211] \downarrow[31]$ & $S_{1}^{0}$ & $\begin{array}{l}E_{2}^{6} \\
E_{2}^{2}\end{array}$ & $\begin{array}{l}E_{2}^{6} \\
S_{1}^{1}\end{array}$ & $\begin{array}{l}E_{2}^{6} \\
S_{1}^{0}\end{array}$ & $S_{1}^{0}$ \\
\hline & $E_{3}^{\prime 4}$ & $E_{3}^{\prime 6}$ & $E_{3}^{\prime 16}$ & $E_{3}^{\prime 6}$ & $E_{3}^{\prime 4}$ \\
\hline
\end{tabular}

Table 5. [4] equivalence class contractions.

\begin{tabular}{ccccc}
\hline contraction & $E_{10}$ & $E_{9}$ & $D_{1} A$ & $K[4]$ \\
\hline$[1111] \downarrow[211]$ & $E_{3}^{\prime 2}$ & $E_{11}^{2}$ & $E_{20}^{4}$ & $E_{3}^{\prime 2}$ \\
& \multicolumn{5}{c}{${E_{3}^{\prime 2}}^{2}$} & $E_{3}^{\prime 2}$ \\
& Stäckel transforms of $V(2)$ \\
\hline$[1111] \downarrow[22]$ & \multicolumn{5}{c}{ Stäckel transforms of $V(2)$} \\
& $E_{3}^{\prime 2}$ & $E_{3}^{\prime 2}$ & $D_{1} C^{2}$ & $D_{3} A^{2}$ \\
\hline$[1111] \downarrow[31]$ & $E_{3}^{\prime 2}$ & $E_{3}^{\prime 2}$ & $E_{3}^{\prime 2}$ & $E_{3}^{\prime 2}$ \\
& $E_{11}^{2}$ & & & \\
\hline$[1111] \downarrow[4]$ & $E_{3}^{\prime 6}$ & $E_{3}^{\prime 6}$ & $E_{3}^{\prime 6}$ & $E_{3}^{\prime 6}$ \\
& $E_{11}^{8}$ & & & \\
& $E_{10}^{6}$ & $E_{10}^{6}$ & $E_{10}^{6}$ & $E_{10}^{6}$ \\
& $E_{9}^{8}$ & & & \\
\hline$[22] \downarrow[4]$ & Stäckel transforms of $V(2)$ \\
\hline$[211] \downarrow[31]$ & $E_{3}^{\prime 1}$ & $E_{3}^{\prime 1}$ & $E_{3}^{\prime-1}$ & $E_{3}^{\prime-1}$ \\
& $E_{3}^{\prime 4}$ & $E_{3}^{\prime 5}$ & $E_{3}^{\prime 4}$ & $E_{3}^{\prime 3}$ \\
& $E_{3}^{\prime 6}$ & $E_{3}^{\prime 6}$ & $E_{3}^{\prime 6}$ & $E_{3}^{\prime 6}$ \\
\hline \multicolumn{5}{c}{}
\end{tabular}

Except for one special class they generalize all of the Helmholtz contractions derived earlier. In particular, contractions of Darboux and Koenigs systems can be described easily. All of the concepts introduced in this paper are clearly also applicable for dimensions $n \geq 3$, see [4]. The conceptual picture is Fig. 1.

The special class that is missing in the present paper is the class of contractions to systems with degenerate Hamiltonians, i.e., systems for which the determinant of the metric tensor is zero. In a paper under preparation we will show that these limits correspond to contractions of $\mathfrak{s o}(4, \mathbb{C})$ to $\mathfrak{e}(3, \mathbb{C})$ and lead to time-dependent conformally superintegrable systems (Schrödinger equations) with potential. We will examine the relations between the contractions in classified in $[8,21]$ and show that they are properly contained in those induced by $\mathfrak{s o}(4, \mathbb{C})$. From Theorem 1.1 we know that the potentials of all Helmholtz superintegrable systems are completely 
Table 6. [0] equivalence class contractions.

\begin{tabular}{ccccccc}
\hline contraction & $E_{20}$ & $E_{11}$ & $E_{3}^{\prime}$ & $D_{1} C$ & $D_{3} A$ & $K[0]$ \\
\hline$[1111] \downarrow[211]$ & $E_{3}^{\prime 2}$ & ${E_{3}^{\prime 2}}^{2}$ & $E_{3}^{\prime 2}$ & $E_{3}^{\prime 2}$ & $E_{3}^{\prime 2}$ & $E_{3}^{\prime 2}$ \\
& $E_{11}^{3}$ & $E_{11}^{3}$ & & $D_{1} C^{3}$ & $D_{1} C^{3}$ & \\
\hline$[1111] \downarrow[22]$ & $E_{11}^{2}$ & $E_{11}^{2}$ & $E_{3}^{\prime 2}$ & $E_{11}^{2}$ & $E_{11}^{2}$ & $E_{11}^{2}$ \\
& & & & & $E_{3}^{\prime 2}$ & $E_{3}^{\prime 2}$ \\
\hline$[1111] \downarrow[31]$ & $E_{3}^{\prime 2}$ & $E_{3}^{\prime 2}$ & $E_{3}^{\prime 2}$ & $E_{3}^{\prime 2}$ & $E_{3}^{\prime 2}$ & $E_{3}^{\prime 2}$ \\
& & & & $D_{1} C^{3}$ & $D_{1} C^{3}$ & \\
\hline$[1111] \downarrow[4]$ & $E_{3}^{\prime 6}$ & $E_{3}^{\prime 6}$ & $E_{3}^{\prime 6}$ & $E_{3}^{\prime 6}$ & $E_{3}^{\prime 6}$ & $E_{3}^{\prime 6}$ \\
& $E_{11}^{8}$ & $E_{11}^{8}$ & & $E_{11}^{8}$ & $E_{11}^{8}$ & \\
\hline$[22] \downarrow[4]$ & $E_{3}^{\prime 4}$ & $E_{3}^{\prime 4}$ & $E_{3}^{\prime 4}$ & $E_{3}^{\prime 4}$ & $E_{3}^{\prime 4}$ & $E_{3}^{\prime 4}$ \\
& $E_{11}^{5}$ & $E_{11}^{5}$ & & $E_{11}^{5}$ & & \\
\hline$[211] \downarrow[31]$ & $E_{3}^{\prime 6}$ & $E_{3}^{\prime 6}$ & $E_{3}^{\prime 6}$ & $E_{3}^{\prime 6}$ & $E_{3}^{\prime 6}$ & $E_{3}^{\prime 6}$ \\
& & & & $D_{1} C^{9}$ & & \\
\hline
\end{tabular}

determined by their free quadratic algebras, i.e., the symmetry algebra that remains when the parameters in the potential are set equal to 0. Thus for classification purposes it is enough to classify free abstract quadratic algebras. We will give a classification of abstract free nondegenerate quadratic algebras and their abstract contractions and discuss which of these abstract systems and contractions correspond to physical systems.

In papers under preparation we will 1) give a precise definition of Böcher contractions and introduce other methods of constructing them, 2) apply the Bôcher construction to degenerate (1-parameter) Helmholtz superintegrable systems (which admit a 1st order symmetry), 3) give a complete classification of free abstract degenerate quadratic algebras and identify which of those correspond to free 2nd order superintegrable systems, 4) classify abstract contractions of degenerate quadratic algebras and identify which of those correspond to geometric contractions of Helmholtz superintegrable systems.

We note that by taking contractions step-by-step from a model of the $S_{9}$ quadratic algebra we can recover the Askey scheme [25]. However, the contraction method is more general. It applies to all special functions that arise from the quantum systems via separation of variables, not just polynomials of hypergeometric type, and it extends to higher dimensions. The functions in the Askey scheme are just those hypergeometric polynomials that arise as the expansion coefficients relating two separable eigenbases that are both of hypergeometric type. Thus, there are some contractions which do not fit in the Askey scheme since the physical system fails to have such a pair of separable eigenbases. In another paper we will analyze the Laplace 2nd order conformally superintegrable systems, determine which of them is exactly solvable or quasi-exactly solvable, identify the spaces of polynomials that arise and examine their behavior under contraction.

\section{Acknowledgements}

This work was partially supported by a grant from the Simons Foundation (\# 208754 to Willard Miller Jr).

\section{References}

[1] Bôcher M., Ueber die Reihenentwickelungen der Potentialtheorie, B.G. Teubner, Leipzig, 1894.

[2] Bromwich T.J.I., Quadratic forms and their classification by means of invariant-factors, Cambridge University Press, Cambridge, 1906. 
[3] Capel J.J., Kress J.M., Invariant classification of second-order conformally flat superintegrable systems, J. Phys. A: Math. Theor. 47 (2014), 495202, 33 pages, arXiv:1406.3136.

[4] Capel J.J., Kress J.M., Post S., Invariant classification and limits of maximally superintegrable systems in 3D, SIGMA 11 (2015), 038, 17 pages, arXiv:1501.06601.

[5] Daskaloyannis C., Tanoudis Y., Quantum superintegrable systems with quadratic integrals on a two dimensional manifold, J. Math. Phys. 48 (2007), 072108, 22 pages, math-ph/0607058.

[6] Evans N.W., Super-integrability of the Winternitz system, Phys. Lett. A 147 (1990), 483-486.

[7] Fordy A.P., Quantum super-integrable systems as exactly solvable models, SIGMA 3 (2007), 025, 10 pages, math-ph/0702048.

[8] Heinonen R., Kalnins E.G., Miller Jr. W., Subag E., Structure relations and Darboux contractions for 2D 2nd order superintegrable systems, SIGMA 11 (2015), 043, 33 pages, arXiv:1502.00128.

[9] Inönü E., Wigner E.P., On the contraction of groups and their representations, Proc. Nat. Acad. Sci. USA 39 (1953), 510-524.

[10] Izmest'ev A.A., Pogosyan G.S., Sissakian A.N., Winternitz P., Contractions of Lie algebras and separation of variables, J. Phys. A: Math. Gen. 29 (1996), 5949-5962.

[11] Izmest'ev A.A., Pogosyan G.S., Sissakian A.N., Winternitz P., Contractions of Lie algebras and the separation of variables: interbase expansions, J. Phys. A: Math. Gen. 34 (2001), 521-554.

[12] Kalnins E.G., Kress J.M., Miller Jr. W., Second-order superintegrable systems in conformally flat spaces. I. Two-dimensional classical structure theory, J. Math. Phys. 46 (2005), 053509, 28 pages.

[13] Kalnins E.G., Kress J.M., Miller Jr. W., Second order superintegrable systems in conformally flat spaces. II. The classical two-dimensional Stäckel transform, J. Math. Phys. 46 (2005), 053510, 15 pages.

[14] Kalnins E.G., Kress J.M., Miller Jr. W., Second order superintegrable systems in conformally flat spaces. III. Three-dimensional classical structure theory, J. Math. Phys. 46 (2005), 103507, 28 pages.

[15] Kalnins E.G., Kress J.M., Miller Jr. W., Second order superintegrable systems in conformally flat spaces. IV. The classical 3D Stäckel transform and 3D classification theory, J. Math. Phys. 47 (2006), 043514, 26 pages.

[16] Kalnins E.G., Kress J.M., Miller Jr. W., Second order superintegrable systems in conformally flat spaces. V. Two- and three-dimensional quantum systems, J. Math. Phys. 47 (2006), 093501, 25 pages.

[17] Kalnins E.G., Kress J.M., Miller Jr. W., Nondegenerate 2D complex Euclidean superintegrable systems and algebraic varieties, J. Phys. A: Math. Theor. 40 (2007), 3399-3411, arXiv:0708.3044.

[18] Kalnins E.G., Kress J.M., Miller Jr. W., Post S., Laplace-type equations as conformal superintegrable systems, Adv. in Appl. Math. 46 (2011), 396-416, arXiv:0908.4316.

[19] Kalnins E.G., Kress J.M., Miller Jr. W., Winternitz P., Superintegrable systems in Darboux spaces, J. Math. Phys. 44 (2003), 5811-5848, math-ph/0307039.

[20] Kalnins E.G., Kress J.M., Pogosyan G.S., Miller Jr. W., Completeness of superintegrability in twodimensional constant-curvature spaces, J. Phys. A: Math. Gen. 34 (2001), 4705-4720, math-ph/0102006.

[21] Kalnins E.G., Miller Jr. W., Quadratic algebra contractions and second-order superintegrable systems, Anal. Appl. (Singap.) 12 (2014), 583-612, arXiv:1401.0830.

[22] Kalnins E.G., Miller Jr. W., Post S., Wilson polynomials and the generic superintegrable system on the 2-sphere, J. Phys. A: Math. Theor. 40 (2007), 11525-11538.

[23] Kalnins E.G., Miller Jr. W., Post S., Models for quadratic algebras associated with second order superintegrable systems in 2D, SIGMA 4 (2008), 008, 21 pages, arXiv:0801.2848.

[24] Kalnins E.G., Miller Jr. W., Post S., Coupling constant metamorphosis and Nth-order symmetries in classical and quantum mechanics, J. Phys. A: Math. Theor. 43 (2010), 035202, 20 pages, arXiv:0908.4393.

[25] Kalnins E.G., Miller Jr. W., Post S., Contractions of 2D 2nd order quantum superintegrable systems and the Askey scheme for hypergeometric orthogonal polynomials, SIGMA 9 (2013), 057, 28 pages, arXiv:1212.4766.

[26] Kalnins E.G., Miller Jr. W., Reid G.J., Separation of variables for complex Riemannian spaces of constant curvature. I. Orthogonal separable coordinates for $\mathrm{S}_{n \mathbf{C}}$ and $\mathrm{E}_{n \mathbf{C}}$, Proc. Roy. Soc. London Ser. A 394 (1984), $183-206$.

[27] Kalnins E.G., Miller Jr. W., Subag E., Bôcher contractions of conformally superintegrable Laplace equations: detailed computations, arXiv:1601.02876. 
[28] Kalnins E.G., Miller Jr. W., Subag E., Laplace equations, conformal superintegrability and Bôcher contractions, Acta Polytechnica, to appear, arXiv:1510.09067.

[29] Koenigs G., Sur les géodésiques a intégrales quadratiques, in Darboux G., Lecons sur la théorie générale des surfaces et les applications geométriques du calcul infinitesimal, Vol. 4, Chelsea, New York, 1972, 368-404.

[30] Kress J.M., Equivalence of superintegrable systems in two dimensions, Phys. Atomic Nuclei 70 (2007), $560-566$.

[31] Miller Jr. W., Li Q., Wilson polynomials/functions and intertwining operators for the generic quantum superintegrable system on the 2-sphere, J. Phys. Conf. Ser. 597 (2015), 012059, 11 pages, arXiv:1411.2112.

[32] Miller Jr. W., Post S., Winternitz P., Classical and quantum superintegrability with applications, J. Phys. A: Math. Theor. 46 (2013), 423001, 97 pages, arXiv:1309.2694.

[33] Nesterenko M., Popovych R., Contractions of low-dimensional Lie algebras, J. Math. Phys. 47 (2006), 123515, 45 pages, math-ph/0608018.

[34] NIST digital library of mathematical functions, available at http://dlmf.nist.gov/.

[35] Post S., Models of quadratic algebras generated by superintegrable systems in 2D, SIGMA 7 (2011), 036, 20 pages, arXiv:1104.0734.

[36] Tempesta P., Turbiner A.V., Winternitz P., Exact solvability of superintegrable systems, J. Math. Phys. 42 (2001), 4248-4257, hep-th/0011209.

[37] Tempesta P., Winternitz P., Harnad J., Miller W., Pogosyan G., Rodriguez M. (Editors), Superintegrability in classical and quantum systems, CRM Proceedings and Lecture Notes, Vol. 37, Amer. Math. Soc., Providence, RI, 2004.

[38] Weimar-Woods E., The three-dimensional real Lie algebras and their contractions, J. Math. Phys. 32 (1991), 2028-2033. 